\title{
A Coordinated Direct AF DF Relay-Aided NOMA Framework for Low Outage
}

This paper was downloaded from TechRxiv (https://www.techrxiv.org).

\section{LICENSE}

CC BY 4.0

SUBMISSION DATE / POSTED DATE

22-05-2021 / 06-12-2021

\section{CITATION}

Jee, Anand; AGRAWAL, KAMAL; Prakriya, Shankar (2021): A Coordinated Direct AF DF Relay-Aided NOMA Framework for Low Outage. TechRxiv. Preprint. https://doi.org/10.36227/techrxiv.14647317.v2

$\mathrm{DOI}$

10.36227/techrxiv.14647317.v2 


\title{
A Coordinated Direct AF/DF Relay-Aided NOMA Framework for Low Outage
}

\author{
Anand Jee, Graduate Student Member, IEEE, Kamal Agrawal, Graduate Student Member, IEEE and \\ Shankar Prakriya, Senior Member, IEEE
}

\begin{abstract}
This paper investigates the performance of a new framework for low-outage downlink non-orthogonal multiple access (NOMA) using a coordinated direct and relay transmission (CDRT) scheme with direct links to both the nearuser (NU) and the far-user (FU). Both $\mathrm{AF}$ and DF relays are considered. In this framework, the NU combines the signals from $B S$ and $R$ at each stage of the successive interference cancellation (SIC) to attain good outage performance. For both $\mathrm{NU}$ and FU, the expressions for outage probability and throughput are derived in closed form. We also derive the highSNR expressions for the outage probability to demonstrate that with the proposed framework, both users harness a diversity of two without feedback bits (this is the only framework to achieve this). We demonstrate that the choice of power allocation coefficient and target symbol rates is crucial to maximizing the NU throughput while ensuring a desired target FU throughput. We demonstrate that CDRT with the proposed framework outperforms known schemes in terms of outage probability, sum throughput, and energy efficiency. Moreover, we also show that optimal rate selection is important to maximize the EE. Monte Carlo simulations validate the accuracy of the derived analytical expressions.
\end{abstract}

Index Terms-Non-orthogonal multiple access (NOMA), coordinated direct and relay transmission (CDRT), energy efficiency (EE), amplify-and-forward (AF), decode-and-forward (DF).

\section{INTRODUCTION}

The proliferation of Internet of Things (IoT) and massive machine-type communication has augmented the demand for higher data rates, low latency, and high bandwidth efficiency in beyond 5G (B5G) and 6G networks [1]-[4]. Multiple access techniques used in older generations of wireless communication networks are insufficient to fulfill these stringent requirements on user density and network traffic. To meet the requirements of future wireless networks, several multiple access technologies were studied in the last decade. Among them, non-orthogonal multiple access (NOMA) has emerged as one of the most promising technologies for future wireless networks due to its high spectral efficiency (SE), low latency, and ability to facilitate massive connectivity [5]-[7].

In contrast to traditional orthogonal multiple access (OMA) schemes, NOMA facilitates concurrent transmission

A. Jee, K. Agrawal, and S. Prakriya are with the Department of Electrical Engineering, Indian Institute of Technology Delhi (IIT Delhi), New Delhi110016, India (e-mail: \{anandjee7, kamal.agrawal, shankar\}@ee.iitd.ac.in)

A portion of this work limited only to AF-NOMA in the absence of BS-FU direct link has been presented in IEEE PIMRC 2021. to multiple users over the same spectral/time/spreading code resource using power domain multiplexing at the transmitter side and successive interference cancellation (SIC) at the receiver side. In downlink NOMA, the user with a poor channel condition is termed as the far user (FU), and the user with a better channel condition is termed as the near user (NU) [8]. The quality of service (QoS) and the coverage area can be further enhanced by using relayed NOMA (R-NOMA) where dedicated relay nodes are used [9]-[15]. In [9], [10], the performance of amplifyand-forward (AF) and decode-and-forward (DF) relaying schemes was compared for different relay selection criteria in an R-NOMA scheme. A novel relay selection strategy was proposed to ensure minimum outage probability in [11]. In [12], the authors analyzed the performance of energy harvesting based R-NOMA assuming that the harvested energy is augmented by regular battery energy. In [13], the effect of imperfect SIC and channel state information (CSI) errors were considered, and performance was analyzed in terms of bit error rate (BER), outage probability, and ergodic capacity. In particular, the authors also demonstrated that with DF relaying, R-NOMA provides better user fairness than cooperative NOMA (C-NOMA). The outage probability of a multi-antenna relay-based hybrid satellite-terrestrial network was investigated in [14]. In [15], the ergodic sumrate performance of the R-NOMA system was analyzed for both AF and DF relaying schemes considering Nakagami-m fading channels.

C-NOMA is another approach to enhance the coverage and QoS in which the NU assists the information transmission to the FU. In [16], considering the direct link to both $\mathrm{NU}$ and FU, the performance of the C-NOMA system was investigated for both half-duplex and full-duplex relaying. Further, in [17] the FU performance was improved by applying OMA/C-NOMA switching in a multiuser scenario, whereas OMA/NOMA/C-NOMA switching with user selection was studied in [18] to improve the QoS in an underlay cognitive radio (CR) NOMA network. Further, in [19], the block error performance for short packet communication in a C-NOMA network was compared to conventional NOMA assuming Rayleigh flat fading channels. To improve the SE, successive user relaying in C-NOMA was discussed in [20].

The use of NOMA in coordinated direct and relay transmission (CDRT) [21-27] is another promising approach to improve the QoS at FU. In a typical CDRT-NOMA 
signalling scheme, the base station (BS) shares a direct link to the $\mathrm{NU}$, while a dedicated relay assists communication to the FU. IoT-assisted CDRT-NOMA was analyzed in terms of outage probability and ergodic sum capacity (ESC) in [21]. In [22], the SE and user fairness index of CDRT-NOMA were analyzed considering perfect and imperfect SIC at NU. Coordinated uplink transmission for the C-NOMA system was studied in [23], and the outage probability was derived considering Nakagami-m fading channels. NOMAbased CDRT and hybrid multiple access (HMA) protocols were studied in [24], and it was shown that NOMA-based CDRT achieves better ESC than HMA but with higher complexity. In [25] a joint downlink-uplink adaptive CDRT based NOMA network was investigated for both perfect and imperfect SIC, and closed-form expressions were derived for the outage probability, ESC, and energy efficiency (EE). Performance of CDRT-NOMA with a direct link to both users was investigated in [26], [28]. In [26], the performance of a DF relay aided CDRT-NOMA was investigated in the presence of a direct link to both the users. In [27], the performance of CDRT-NOMA was investigated with multiple FUs in terms of ergodic capacity and outage probability. However, the absence of R-NU and BS-FU links limits the performance of both $\mathrm{NU}$ and $\mathrm{FU}^{1}[28]$ investigated the performance of fixed-gain AF relay-based CDRT-NOMA in the presence of the BS-FU link assuming sub-optimal selection combining at the FU. A detailed comparison of our work with existing literature on C-NOMA, R-NOMA, and CDRT-NOMA is provided in Table [

\section{A. Motivation and Contribution}

In CDRT-NOMA, signalling occurs in two phases. In the first phase, BS broadcasts the superimposed symbols to the $\mathrm{NU}$ and the relay node, and NU decodes its own symbols after decoding and removing the FU's symbols using SIC. In the second phase, BS transmits a new set of symbols to $\mathrm{NU}$, and the relay node forwards the decoded symbols to FU, which causes interference at NU. However, based on the decoding status of the FU's symbols in the first phase, NU can perform the interference cancellation. Existing CDRTNOMA systems have the following limitations:

- The performance of NU depends a lot on the decoding status of the symbols in the first phase. If the BS-NU link fails in the first phase, then NU will not be able to decode the desired symbols together with the FU's symbols, and thus, the interference in the second phase cannot be canceled. Therefore, the decoding status in the first phase is a bottleneck for the NU's performance.

- The relay node has to decode the FU's symbols successfully to assist the information transmission to FU

\footnotetext{
${ }^{1}$ Unlike [27], in this work, we do not consider multiple FUs as this may result in increased complexity at the $\mathrm{NU}$ receiver due to requirement of multiple SIC with combining at each stage. Note that multiple SIC will lead to SIC failure in some realizations, in which case NU might fail to decode some FU symbols and hence might lose in performance due to participation in NOMA signalling, which is contrary to the objective of this paper.
}

in the second phase. If the BS-R link goes into a deep fade, the FU will be in outage.

It is evident from the above that NU loses on performance due to participation in NOMA signalling. Also, the absence of the BS-FU direct link limits performance.

Due to participation in NOMA signalling, NU needs to decode the FU symbol first to perform SIC (complexity of the NU receiver clearly increases) before it can decode its own symbol. Depending on the channel condition, target rate, and power allocation factor, NU might occasionally fail to decode its own symbol due to insufficient signal-to-noise ratio (SNR) or unsuccessful decoding of the FU symbol. In such a scenario, the BS has to re-transmit the superimposed symbol, which causes a reduction in SE and EE. In CNOMA, NU incurs a loss in SE (due to allocation of power to the FU symbols and the use of two signalling phases) and EE as well (it is required to expend energy for relaying the FU symbol). To date, virtually all existing literature on CDRT-NOMA focuses on AF and DF relaying in the absence of the BS-FU direct link. The availability of the BS-FU direct link enhances the QoS at FU and lowers the required energy consumption at the relay. In [26], DF relayaided CDRT-NOMA in the presence of the BS-FU link has been investigated for three different cases a) fixed relaying where the DF relay always forwards the incoming signal from BS, b) selective DF where the relay only forwards after successful decoding of the incoming signal and requires two-bit feedback, and c) incremental selective DF relaying where the relayed link is used opportunistically and requires three-bit feedback. In particular, for the lower analytical complexity, the power allocation at the relay is considered such that after linearly combining the first and second phase signals, the FU symbols are canceled at the NU, and both the users recover their desired symbols. This is sub-optimal, and results in a diversity loss for both the users.

All the aforementioned works have laid a solid foundation for the use of C-NOMA and CDRT to improve the FU's performance, but there has been little effort to improve the NU's performance. It might not be possible to satisfy NU's constraints using these CDRT approaches (they only guarantee a diversity of one at the NU [29]). However, considering an AF or DF relay to assist FU and NU with optimal combining at both users improves the NU diversity. It results in improved QoS for both users. The NU will combine with SIC (in a framework discussed in this paper) but attains much better performance, which is necessary for many scenarios like multimedia and gaming applications that impose a low outage and higher link reliability requirements. We also discuss the optimization of NU performance while ensuring the desired performance at FU. The novel contributions of this paper are as follows:

- We investigate the performance of a new framework for CDRT based downlink NOMA network with the direct link to both NU and FU. The NU combines the incoming signals from BS and the relay at each stage of the SIC to enhance its performance. This 
TABLE I: Comparison of literature on C-NOMA, R-NOMA, CDRT-NOMA with our work. $N_{r}$ and $N_{t}$ represent the number of transmit and receive antennas, respectively, while $m$ stands for the $m^{\text {th }}$ user and $\mu$ denotes the shape parameter for Nakagami- $\mu$ distribution.

\begin{tabular}{|c|c|c|c|c|c|c|c|c|c|c|c|c|c|c|c|}
\hline & {$[16$} & 24 & 25 & 22 & 113 & 14 & 15 & 21 & 20 & [19 & 23 & 28 & 26 & 27. & Our Work \\
\hline Relayed NOMA & $x$ & $\bar{x}$ & $\bar{x}$ & $\bar{x}$ & $\sqrt{\checkmark}$ & $\checkmark$ & $\bar{\checkmark}$ & $\bar{x}$ & $\bar{x}$ & $\bar{x}$ & $\bar{x}$ & $x$ & $x$ & $\bar{x}$ & $x$ \\
\hline Cooperative NOMA & $\checkmark$ & $\times$ & $x$ & $x$ & $x$ & $x$ & $x$ & $x$ & $\checkmark$ & $\checkmark$ & $x$ & $x$ & $x$ & $x$ & $x$ \\
\hline CDRT & $x$ & $\checkmark$ & $\checkmark$ & $\checkmark$ & $x$ & $x$ & $x$ & $\checkmark$ & $x$ & $\times$ & $\checkmark$ & $\checkmark$ & $\checkmark$ & $\checkmark$ & $\checkmark$ \\
\hline AF relaying & $x$ & $x$ & $x$ & $x$ & $x$ & $x$ & $\checkmark$ & $\times$ & $x$ & $x$ & $x$ & $\checkmark$ & $x$ & $\times$ & $\checkmark$ \\
\hline DF relaying & $\checkmark$ & $\checkmark$ & $\checkmark$ & $\checkmark$ & $\checkmark$ & $\checkmark$ & $\checkmark$ & $\checkmark$ & $\checkmark$ & $\checkmark$ & $\checkmark$ & $x$ & $\checkmark$ & $\checkmark$ & $\checkmark$ \\
\hline Rayleigh fading & $\checkmark$ & $x$ & $\checkmark$ & $x$ & $\checkmark$ & $\checkmark$ & $x$ & $\checkmark$ & $\checkmark$ & $\checkmark$ & $x$ & $x$ & $\checkmark$ & $\checkmark$ & $\checkmark$ \\
\hline Nakagami-m fading & $x$ & $\checkmark$ & $x$ & $\checkmark$ & $x$ & $\checkmark$ & $\checkmark$ & $\times$ & $x$ & $x$ & $\checkmark$ & $\checkmark$ & $\times$ & $x$ & $x$ \\
\hline Direct link to FU & $\checkmark$ & $x$ & $x$ & $x$ & $x$ & $x$ & $x$ & $\times$ & $x$ & $\checkmark$ & $\checkmark$ & $\checkmark$ & $\checkmark$ & $x$ & $\checkmark$ \\
\hline Direct link to NU & $x$ & $x$ & $x$ & $\checkmark$ & $x$ & $\checkmark$ & $x$ & $\checkmark$ & $\checkmark$ & $\checkmark$ & $\checkmark$ & $\checkmark$ & $\checkmark$ & $\checkmark$ & $\checkmark$ \\
\hline Optimal combining at FU & $\checkmark$ & $x$ & $x$ & $\checkmark$ & $x$ & $x$ & $x$ & $\times$ & $x$ & $\checkmark$ & $x$ & $x$ & $x$ & $x$ & $\checkmark$ \\
\hline Optimal combining at NU & $x$ & $\times$ & $x$ & $x$ & $x$ & $x$ & $x$ & $\times$ & $x$ & $\times$ & $\times$ & $x$ & $x$ & $x$ & $\checkmark$ \\
\hline Energy Efficiency & $\checkmark$ & $x$ & $\checkmark$ & $\checkmark$ & $x$ & $x$ & $x$ & $\times$ & $x$ & $x$ & $x$ & $x$ & $x$ & $\times$ & $\checkmark$ \\
\hline Outage and/or throughput & $\checkmark$ & $\times$ & $\checkmark$ & $x$ & $\checkmark$ & $\checkmark$ & $\checkmark$ & $\checkmark$ & $\checkmark$ & $\times$ & $\checkmark$ & $\checkmark$ & $\checkmark$ & $\checkmark$ & $\checkmark$ \\
\hline BER/SER/BLER/Capacity & $x$ & $\checkmark$ & $\checkmark$ & $\checkmark$ & $\checkmark$ & $x$ & $\checkmark$ & $\checkmark$ & $x$ & $\checkmark$ & $x$ & $x$ & $x$ & $\checkmark$ & $x$ \\
\hline Power allocation at Source & $\checkmark$ & $\checkmark$ & $\checkmark$ & $\checkmark$ & $\checkmark$ & $\checkmark$ & $\checkmark$ & $\checkmark$ & $\checkmark$ & $\checkmark$ & $\checkmark$ & $\checkmark$ & $\checkmark$ & $\checkmark$ & $\checkmark$ \\
\hline Power allocation at Relay & - & $\checkmark$ & - & $\checkmark$ & $\checkmark$ & $x$ & $\checkmark$ & $\times$ & $x$ & $\times$ & $\checkmark$ & $x$ & $\checkmark$ & $\checkmark$ & $\checkmark$ \\
\hline Independent $\alpha_{B}$ and $\alpha_{R}$ & - & $\checkmark$ & - & $\checkmark$ & $\checkmark$ & $\times$ & $x$ & $\times$ & $x$ & $x$ & $x$ & $x$ & $x$ & $\times$ & $\checkmark$ \\
\hline$\alpha_{B}<\left(1-\alpha_{B}\right)$ or $\alpha_{R}<\left(1-\alpha_{R}\right)$ & $\checkmark$ & $\checkmark$ & $\checkmark$ & $\checkmark$ & $x$ & $\checkmark$ & $\checkmark$ & $\checkmark$ & $x$ & $\checkmark$ & $x$ & $\checkmark$ & $x$ & $\checkmark$ & $x$ \\
\hline Optimization of $\alpha_{B}$ & $x$ & $x$ & $\checkmark$ & $x$ & $x$ & $x$ & $x$ & $\times$ & $\checkmark$ & $\times$ & $x$ & $x$ & $x$ & $x$ & $\checkmark$ \\
\hline$\tau_{N}^{\max }$ while ensuring fixed $\tau_{F}$ & $x$ & $x$ & $\times$ & $x$ & $x$ & $x$ & $x$ & $\times$ & $x$ & $\bar{x}$ & $x$ & $x$ & $\bar{x}$ & $\times$ & $\checkmark$ \\
\hline Reward NU for cooperation & $\times$ & $x$ & $x$ & $x$ & $x$ & $x$ & $x$ & $\times$ & $x$ & $x$ & $x$ & $x$ & $x$ & $x$ & $\checkmark$ \\
\hline Diversity order at NU & 0 or 1 & - & 0 or 1 & - & - & 2 & - & - & - & 1 & 0 & $\mathrm{~m} \mu$ & 1 or 2 & $x$ & 2 \\
\hline Diversity order at FU & $0 / 1 / 2$ & - & 0 or 1 & - & - & $\max \left(N_{r}, N_{t}\right)$ & - & - & - & 2 & 0 & $\mathrm{~m} \mu$ & 1 or 2 & $\times$ & 2 \\
\hline Feedback bits required & - & - & - & - & - & - & - & - & - & - & - & - & 1 or 3 & $\times$ & - \\
\hline
\end{tabular}

incentivizes its participation in NOMA signalling. The FU also uses a combination of direct and relayed signals. Considering both AF and DF relaying, we present closed-form expressions for the outage probability and the throughput of both $\mathrm{NU}$ and FU with this new framework. Unlike other works [30], [31] on AF CDRT NOMA where (for analytical simplicity) the harmonic to min approximation is used, we derive the exact outage probability in closed form. The case when the BS-FU link is absent follows as a special case.

- We also derive highly accurate high-SNR expressions for the outage probability to demonstrate that combining direct and relayed signals at $\mathrm{NU}$ as well as FU (while performing SIC at NU) allows both NU and FU to harness a diversity of 2 with both $\mathrm{AF}$ and DF relaying. It is emphasized that this is the only framework to achieve this without feedback bits or incremental signalling. Clearly, the NU is rewarded for participation in NOMA signalling. However, in the absence of the BS-FU link, FU attains a diversity of only one.

- We show that how the choice of power allocation coefficients and target symbol rates are crucial to maximizing the NU throughput while guaranteeing the desired throughput at FU. Due to the intelligent combining in different stages of the SIC at the NU, the power allocated to the NU symbols can be decreased, and that allocated power to the FU symbols can be increased, which ensures desired QoS at a more distant FU. We also present an approximate closed-form expression for the power allocation coefficient that maximizes NU throughput for a given FU throughput requirement. In addition to this, using the derived expressions for the optimum power allocation coefficients, an NU throughput maximization problem is formulated to determine the optimal NU and FU target rate pair.

- Further, we demonstrate that the proposed framework with CDRT (without using any feedback) always outperforms SDF CDRT-NOMA [26] (which uses two feedback bits) in terms of outage probability, sum throughput, and energy efficiency (EE) by a huge margin and also achieves better diversity at both the users. The additional complexity required for combining signals at the NU is very small.

- Moreover, we compare the performance of the proposed CDRT-NOMA framework to its OMA counterpart (with relaying) and observe that the proposed framework ensures higher EE, which is an important outcome of this paper. We further observe that the availability of the BS-FU link helps achieve a higher EE compared to 
the case when the BS-FU link is absent. Also, optimal rate selection is important to maximize the EE.

The rest of this paper is structured as follows. Section $\Pi$ elaborates on the system model for AF as well as the DF relay-assisted CDRT-NOMA framework. Section III analyzes the performance with the proposed framework in terms of outage probability. Section [V] discusses NU throughput and EE maximization. Numerical results based on mathematical analysis are compared to computer simulations in Section V] Finally, Section VI concludes this paper.

Notations: $\mathcal{C} \mathcal{N}\left(0, \sigma^{2}\right)$ represents a zero-mean complex Gaussian distribution with variance $\sigma^{2} . f_{X}(x)$ and $U(\cdot)$ respectively denote the probability density function (PDF) of a random variable $(\mathrm{RV}) X$ and the unit step function. $\mathcal{E}_{1}(\cdot)$, $K_{1}(\cdot, \cdot)$ and $\Gamma(a, x ; b)$ denote the exponential integral of type 1 , the modified Bessel function of the second kind and the generalized incomplete gamma function, respectively.

\section{System MODEL}

As depicted in Fig. 1, we consider a CDRT downlink NOMA framework consisting of a base station $\mathrm{B}$, a NU $\mathrm{U}_{\mathrm{N}}$, a FU $\mathrm{U}_{\mathrm{F}}$, and a relay station $\mathrm{R}$. Both $\mathrm{U}_{\mathrm{N}}$ and $\mathrm{U}_{\mathrm{F}}$ have stringent outage QoS constraints. All nodes operate in the half-duplex mode and are equipped with a single antenna. Communication to $\mathrm{U}_{\mathrm{N}}$ and $\mathrm{U}_{\mathrm{F}}$ takes place in two signalling phases. In the first phase, $\mathrm{B}$ communicates to $\mathrm{U}_{\mathrm{N}}, \mathrm{R}$, and $\mathrm{U}_{\mathrm{F}}$ over direct links, while in the second phase, communication from $\mathrm{R}$ to $\mathrm{U}_{\mathrm{N}}$ and $\mathrm{U}_{\mathrm{F}}$ take place using either $\mathrm{AF}$ or $\mathrm{DF}$ mode of relaying.

The channel coefficients $h_{i j} \sim \mathcal{C} \mathcal{N}\left(0,1 / \lambda_{i j}\right)$ with $i \in$ $\{B, R\}, j \in\{R, N, F\}$ are assumed to be independent and of quasi-static Rayleigh fading type, where $\lambda_{i j}=d_{i j}^{m}$, and $m$ is the path-loss exponent. The additive zero-mean complex Gaussian noise at all the receiving nodes is assumed to be of variance $\sigma^{2}$. Superscript "I" and "II" are used to represent first and second phase quantities.

\section{A. AF Relaying}

In the first phase, B transmits a superposition of unitenergy symbols $s_{N}$ and $s_{F}$ (of information rates $R_{N}$ and $R_{F}$ ) intended respectively for $\mathrm{U}_{\mathrm{N}}$ and $\mathrm{U}_{\mathrm{F}}$. The transmit power $P_{B}$ is apportioned to $\mathrm{U}_{\mathrm{N}}$ and $\mathrm{U}_{\mathrm{F}}$ symbols in the ratio $\alpha_{B}$ : $(1-$ $\left.\alpha_{B}\right)$. Thus, the superimposed symbol can be expressed as $s_{B}=\sqrt{P_{B} \alpha_{B}} s_{N}+\sqrt{P_{B}\left(1-\alpha_{B}\right)} s_{F}$ [32]. The sampled matched filter outputs at $\mathrm{U}_{\mathrm{N}}, \mathrm{R}$ and $\mathrm{U}_{\mathrm{F}}$ are

$$
\begin{aligned}
& y_{N}^{I}=s_{B} h_{B N}+w_{N}^{I}, \\
& y_{R}^{I}=s_{B} h_{B R}+w_{R}^{I}, \\
& y_{F}^{I}=s_{B} h_{B F}+w_{F}^{I},
\end{aligned}
$$

respectively, wherein $w_{N}^{I}, w_{R}^{I}$ and $w_{F}^{I}$ are the respective additive Gaussian noise samples. In accordance with the concept of NOMA, $\mathrm{U}_{\mathrm{N}}$ first decodes the FU symbol $s_{F}$, and then performs SIC to decode the NU symbol $s_{N}$ [33].
Using (1), the SINR $\Gamma_{N F}^{I}$ to decode $s_{F}$, and the SNR $\Gamma_{N}^{I}$ to decode $s_{N}$ at $\mathrm{U}_{\mathrm{N}}$ after $\mathrm{SIC}$, are expressed as

$$
\begin{aligned}
& \Gamma_{N F}^{I}=\frac{\left(1-\alpha_{B}\right) \rho_{B}\left|h_{B N}\right|^{2}}{\alpha_{B} \rho_{B}\left|h_{B N}\right|^{2}+1} \\
& \Gamma_{N N}^{I}=\alpha_{B} \rho_{B}\left|h_{B N}\right|^{2}, \quad \text { provided } \Gamma_{N F}^{I} \geq \gamma_{F},
\end{aligned}
$$

where $\rho_{B}=P_{B} / \sigma^{2}$ represents the transmit $\mathrm{SNR}$ at $\mathrm{B}$ and $\gamma_{F}=2^{R_{F}}-1$ represents the threshold SNR at $\mathrm{U}_{\mathrm{F}}$. Using (3), the SINR $\Gamma_{F F}^{I}$ to decode $s_{F}$ at $\mathrm{U}_{\mathrm{F}}$ can be expressed as

$$
\Gamma_{F F}^{I}=\frac{\left(1-\alpha_{B}\right) \rho_{B}\left|h_{B F}\right|^{2}}{\alpha_{B} \rho_{B}\left|h_{B F}\right|^{2}+1} .
$$

Let $P_{R}$ denote the available transmit power at $\mathrm{R}$. In the second phase, $\mathrm{R}$ amplifies the incoming signal from the first phase and then forwards $s_{R}=\beta y_{R}^{I}$ to $\mathrm{U}_{\mathrm{N}}$ and $\mathrm{U}_{\mathrm{F}}$ as shown in Fig 1 (b), where $\beta=\sqrt{\frac{\rho_{R}}{\rho_{B}\left|h_{B R}\right|^{2}+1}}$ with $\rho_{R}=\frac{P_{R}}{\sigma^{2}}$. The signals received at $\mathrm{U}_{\mathrm{N}}$ and $\mathrm{U}_{\mathrm{F}}$ in the second phase are given by

$$
\begin{aligned}
& y_{N}^{I I}=\beta y_{R}^{I} h_{R N}+w_{N}^{I I}, \\
& y_{F}^{I I}=\beta y_{R}^{I} h_{R F}+w_{F}^{I I},
\end{aligned}
$$

respectively, where $w_{N}^{I I}$ and $w_{F}^{I I}$ are the additive noise samples at $U_{N}$ and $U_{F}$. Similar to the first phase, using (7) the SINRs $\Gamma_{N F}^{I I}$ and $\Gamma_{N N}^{I I}$ to decode $s_{F}$ and $s_{N}$ (after SIC) are expressed as

$$
\begin{aligned}
\Gamma_{N F}^{I I} & =\frac{\rho_{B} \beta^{2}\left(1-\alpha_{B}\right)\left|h_{B R}\right|^{2}\left|h_{R N}\right|^{2}}{\rho_{B} \beta^{2} \alpha_{B}\left|h_{B R}\right|^{2}\left|h_{R N}\right|^{2}+\left|h_{R N}\right|^{2} \beta^{2}+1} \\
& =\frac{\rho_{B} \rho_{R}\left(1-\alpha_{B}\right)\left|h_{B R}\right|^{2}\left|h_{R N}\right|^{2}}{\rho_{B} \rho_{R} \alpha_{B}\left|h_{B R}\right|^{2}\left|h_{R N}\right|^{2}+\rho_{R}\left|h_{R N}\right|^{2}+\rho_{B}\left|h_{B R}\right|^{2}+1} \\
\Gamma_{N N}^{I I} & =\frac{\rho_{B} \beta^{2} \alpha_{B}\left|h_{B R}\right|^{2}\left|h_{R N}\right|^{2}}{\beta^{2}\left|h_{R N}\right|^{2}+1} \\
& =\frac{\rho_{B} \rho_{R} \alpha_{B}\left|h_{B R}\right|^{2}\left|h_{R N}\right|^{2}}{\rho_{R}\left|h_{R N}\right|^{2}+\rho_{B}\left|h_{B R}\right|^{2}+1}, \quad \text { provided } \Gamma_{N F}^{I I}>\gamma_{F} .
\end{aligned}
$$

Using (8), the SINR to decode $s_{F}$ at $\mathrm{U}_{\mathrm{F}}$ is given by

$$
\begin{aligned}
\Gamma_{F F}^{I I} & =\frac{\rho_{B} \beta^{2}\left(1-\alpha_{B}\right)\left|h_{B R}\right|^{2}\left|h_{R F}\right|^{2}}{\rho_{B} \beta^{2} \alpha_{B}\left|h_{B R}\right|^{2}\left|h_{R F}\right|^{2}+\left|h_{R F}\right|^{2} \beta^{2}+1} \\
& =\frac{\rho_{B} \rho_{R}\left(1-\alpha_{B}\right)\left|h_{B R}\right|^{2}\left|h_{R F}\right|^{2}}{\rho_{B} \rho_{R} \alpha_{B}\left|h_{B R}\right|^{2}\left|h_{R F}\right|^{2}+\rho_{R}\left|h_{R F}\right|^{2}+\rho_{B}\left|h_{B R}\right|^{2}+1} .
\end{aligned}
$$

The signals from the first and second phases are combined at $\mathrm{U}_{\mathrm{F}}$. This paper uses an approach that deviates from all existing works to date and enables $U_{N}$ to combine the signals in the two phases at each stage of the SIC. We show that this enables $U_{N}$ to attain very good performance and harness a diversity of two without any feedback. This allows the network to be used for multimedia and other applications that impose strict outage QoS constraints. It is emphasized that none of the techniques suggested so far [26], [29] can ensure a diversity of two at the NU in the absence of any feedback ${ }^{2}$. This improvement in diversity and

\footnotetext{
${ }^{2}$ Since the suggested combining based SIC at $\mathrm{U}_{\mathrm{N}}$ improves performance when the direct links to $U_{N}$ fails, the performance advantage is expected to be retained in the case when feedback is available.
} 


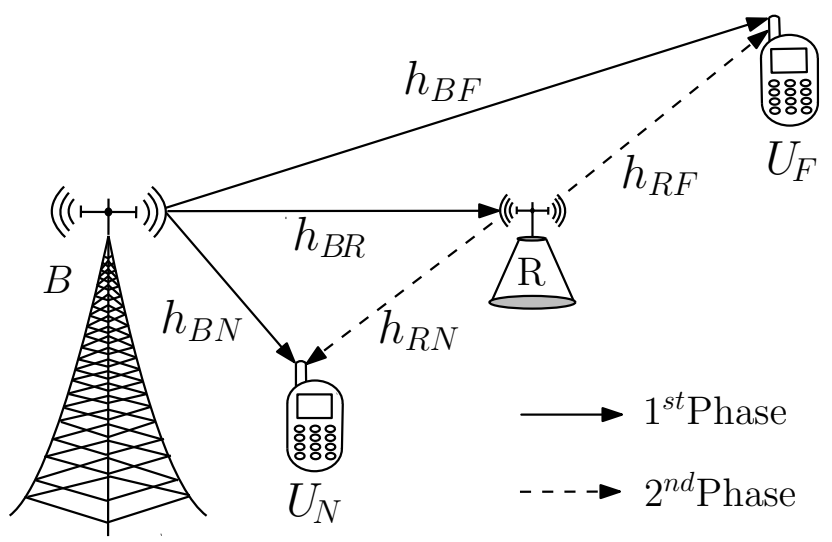

(a)

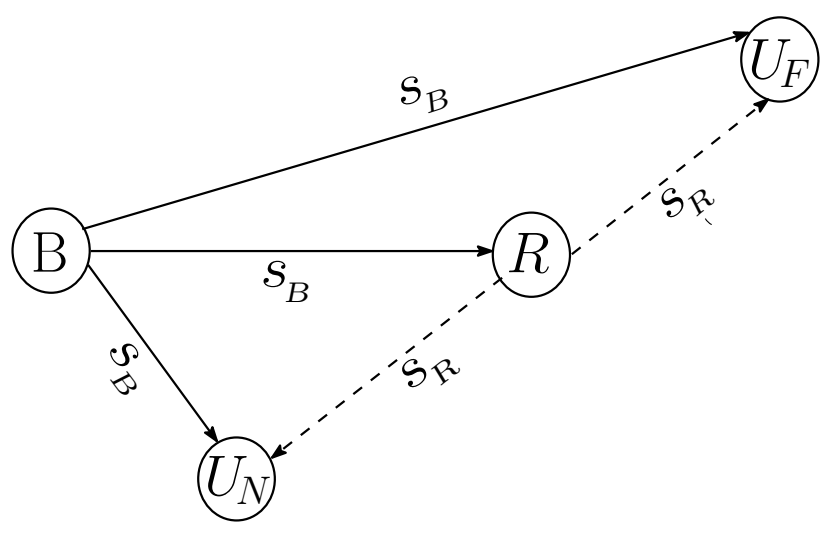

(b)

Fig. 1: (a) System Model. (b) An illustration of the proposed CDRT transmission process, where $s_{B}$ $=\sqrt{P_{B} \alpha_{B}} s_{N}+\sqrt{P_{B}\left(1-\alpha_{B}\right)} s_{F}$. Further, in case of AF relaying $s_{R}=\beta\left(h_{B R}\left(\sqrt{P_{B} \alpha_{B}} s_{N}+\sqrt{P_{B}\left(1-\alpha_{B}\right)} s_{F}\right)+w_{R}^{I}\right)$, and in case of DF relaying $s_{R}=\sqrt{P_{R} \alpha_{R}} s_{N}+\sqrt{P_{R}\left(1-\alpha_{R}\right)} s_{F}$ if $\Gamma_{R F}^{I} \geq \gamma_{F}, \Gamma_{R N}^{I} \geq \gamma_{N}$ and $s_{R}=\sqrt{P_{R}} s_{F}$ if $\Gamma_{R F}^{I}>\gamma_{F}, \Gamma_{R N}^{I}<\gamma_{N}$.

throughput performance also serves as an incentive for $\mathrm{U}_{\mathrm{N}}$ to participate in NOMA signalling. Clearly, $\mathrm{U}_{\mathrm{N}}$ cannot attain this performance using traditional OMA. In this respect, this work is quite different from all existing works.

$\mathrm{U}_{\mathrm{F}}$ combines $y_{F}^{I}$ and $y_{F}^{I I}$ to decode $s_{F}$ with SINR $\Gamma_{F F}^{C O M}=$ $\Gamma_{F F}^{I}+\Gamma_{F F}^{I I}$. If $s_{F}$ is decoded successfully in the first phase, $\mathrm{U}_{\mathrm{N}}$ cancels the interference from $y_{N}^{I I}$ and then combines the first and second phase signals to decode $s_{N}$ with SNR $\Gamma_{N N}^{C O M}=\Gamma_{N N}^{I}+\Gamma_{N N}^{I I}$. However, when $\mathrm{U}_{\mathrm{N}}$ fails to decode $s_{F}$, it combines $y_{N}^{I}$ and $y_{N}^{I I}$ to first decode $s_{F}$ with SINR $\Gamma_{N F}^{C O M}=\Gamma_{N F}^{I}+\Gamma_{N F}^{I I}$, cancels interference, and then decodes $s_{N}$ with SNR $\Gamma_{N N}^{C O M}=\Gamma_{N N}^{I}+\Gamma_{N N}^{I I}$.

\section{B. DF relaying}

As in AF relaying, B transmits the superposed signal $s_{B}$ in the first phase. Therefore, the SINRs $\Gamma_{N F}^{I}, \Gamma_{N N}^{I}$ and $\Gamma_{F}^{I}$ remains same. Different from AF relaying, the DF relay decodes $s_{F}$ and $s_{N}$ by implementing SIC. The SINRs $\Gamma_{R F}^{I}$ and $\Gamma_{R N}^{I}$ (after SIC) to decode $s_{F}$ and $s_{N}$ at R can be expressed as

$$
\begin{aligned}
& \Gamma_{R F}^{I}=\frac{\left(1-\alpha_{B}\right) \rho_{B}\left|h_{B R}\right|^{2}}{\alpha_{B} \rho_{B}\left|h_{B R}\right|^{2}+1}, \\
& \Gamma_{R N}^{I}=\alpha_{B} \rho_{B}\left|h_{B R}\right|^{2} \text { provided } \Gamma_{R F}^{I} \geq \gamma_{F} .
\end{aligned}
$$

In the second phase, based on the decoding status of $s_{N}$ and $s_{F}$, R transmits either superimposed signal $s_{R}$ or only the FU symbol $s_{F}$ as shown in Fig 1 (b). If $\mathrm{R}$ decodes both $s_{N}$ and $s_{F}$ successfully ${ }^{3}$ then it forwards the superimposed signal $s_{R}=\sqrt{P_{R} \alpha_{R} s_{N}+\sqrt{P_{R}\left(1-\alpha_{R}\right)} s_{F}}$ to both $\mathrm{U}_{\mathrm{N}}$ and $\mathrm{U}_{\mathrm{F}}$, where $\alpha_{R}$ and $\left(1-\alpha_{R}\right)$ denotes the portion of power allocated to $U_{N}$ and $U_{F}$, respectively. Note that DF relaying (unlike its AF counter part) allows different NOMA power allocations at $\mathrm{B}$ and $\mathrm{R}$. The signals received

\footnotetext{
${ }^{3}$ In CDRT-NOMA with DF relaying, if R successfully decodes $s_{N}$ and $s_{F}$, the power allocation takes place at both $\mathrm{B}$ and $\mathrm{R}$, thus, DF provides more flexibility and a performance improvement can be expected in comparison to $\mathrm{AF}$ relaying.
}

at $\mathrm{U}_{\mathrm{N}}$ and $\mathrm{U}_{\mathrm{F}}$ can be expressed as $y_{N-S}^{I I}=s_{R} h_{R N}+w_{N}^{I I}$ and $y_{F-S}^{I I}=S_{R} h_{R F}+w_{F}^{I I}$, where $-S$ in the subscript is used to emphasize that a superposed signal is transmitted by $\mathrm{R}$. Using $y_{N-S}^{I I}$, the respective SINRs before and after SIC $\left(\Gamma_{N F-S}^{I I}\right.$ and $\left.\Gamma_{N N-S}^{I I}\right)$ to decode $s_{F}$ and $s_{N}$ at $\mathrm{U}_{\mathrm{N}}$ are

$$
\begin{aligned}
& \Gamma_{N F-S}^{I I}=\frac{\left(1-\alpha_{R}\right) \rho_{R}\left|h_{R N}\right|^{2}}{\alpha_{R} \rho_{R}\left|h_{R N}\right|^{2}+1}, \\
& \Gamma_{N N-S}^{I I}=\alpha_{R} \rho_{R}\left|h_{R N}\right|^{2} \quad \text { provided } \Gamma_{N F-S}^{I I} \geq \gamma_{F} .
\end{aligned}
$$

Using $y_{F-S}^{I I}$, the SINR $\Gamma_{F F-S}^{I I}$ at $\mathrm{F}$ to decode $s_{F}$ is

$$
\Gamma_{F F-S}^{I I}=\frac{\left(1-\alpha_{R}\right) \rho_{R}\left|h_{R F}\right|^{2}}{\alpha_{R} \rho_{R}\left|h_{R F}\right|^{2}+1} .
$$

However, if $\mathrm{R}$ fails to decode $s_{N}$ then it forwards only $s_{F}$. Now, the received signals at $\mathrm{U}_{\mathrm{N}}$ and $\mathrm{U}_{\mathrm{F}}$ are expressed as $y_{N-F}^{I I}=\sqrt{P_{R}} s_{F} h_{R N}+w_{N}^{I I}$ and $y_{F-F}^{I I}=\sqrt{P_{R}} S_{F} h_{R F}+w_{F}^{I I}$ respectively, where the subscript $-F$ is used to emphasize that $\mathrm{R}$ merely transmits $s_{F}$. Using $y_{N-F}^{I I}$ and $y_{F-F}^{I I}$, the respective SINRs $\Gamma_{N F-F}^{I I}$ and $\Gamma_{F F-F}^{I I}$ to decode $s_{F}$ at $\mathrm{U}_{\mathrm{N}}$ and $\mathrm{U}_{\mathrm{F}}$ can be expressed as

$$
\begin{aligned}
& \Gamma_{N F-F}^{I I}=\rho_{R}\left|h_{R N}\right|^{2}, \\
& \Gamma_{F F-F}^{I I}=\rho_{R}\left|h_{R F}\right|^{2} .
\end{aligned}
$$

$\mathrm{U}_{\mathrm{F}}$ combines $y_{F}^{I}$ with $y_{F-S}^{I I}$ when $s_{N}$ and $s_{F}$ are decoded at $\mathrm{R}$, or with $y_{F-F}^{I I}$ otherwise. The combined SINRs are $\Gamma_{F F-S}^{C O M}=\Gamma_{F F}^{I}+\Gamma_{F F-S}^{I I}$ or $\Gamma_{F F-F}^{C O M}=\Gamma_{F F}^{I}+\Gamma_{F F-F}^{I I}$ respectively. However, if $s_{F}$ is decoded successfully in the first phase, $\mathrm{U}_{\mathrm{N}}$ cancels interference from $y_{N-S}^{I I}$ or $y_{N-F}^{I I}$ and then combines the first and second phase signals to decode $s_{N}$ with SNR $\Gamma_{N N-S}^{C O M}=\Gamma_{N N}^{I}+\Gamma_{N N-S}^{I I}$ or $\Gamma_{N N}^{I}$. When $\mathrm{U}_{\mathrm{N}}$ fails to decode $s_{F}$, it combines $y_{N}^{I}$ and $y_{N-S}^{N I}$ or $y_{N-F}^{I I}$ to first decode $s_{F}$ with SINR $\Gamma_{N F-S}^{C O M}=\Gamma_{N F}^{I}+\Gamma_{N F-S}^{I I}$ or $\Gamma_{N F-F}^{C O M}=\Gamma_{N F}^{I}+\Gamma_{N F-F}^{I I}$. Subsequently, after cancelling the interference due to $s_{F}$, decoding of $s_{N}$ takes place with SNR $\Gamma_{N N-S}^{C O M}=\Gamma_{N N}^{I}+\Gamma_{N N-S}^{I I}$ or $\Gamma_{N N}^{I}$. In this paper, we compare the performance of the 
proposed framework with a relayed OMA scheme described below.

\section{Relayed OMA Scheme}

In the considered relayed OMA system, B transmits $x_{F}$ to $U_{F}, U_{N}$, and $R$ in the first signalling phase. In the second signalling phase, $\mathrm{R}$ forwards either the amplified (with AF relaying) or the decoded (with DF relaying) FU symbol, while B transmits $x_{N}$ to NU. Using the decoded FU symbol in the first phase, NU cancels the interference caused by transmission by $\mathrm{R}$ before decoding. However, if NU fails to decode $x_{F}$ in the first phase, then the signal received from $\mathrm{R}$ in the second phase acts as interference at $\mathrm{U}_{\mathrm{N}}$ and $x_{N}$ transmitted from B acts as interference at FU. Note that NU receives symbols in both time-slots in this OMA scheme with relaying. It can therefore be expected to attain high throughput. We observe in Section $\mathrm{V}$ that the proposed framework yields much better throughput performance than its relayed OMA counterpart.

\section{Outage Probability Analysis}

In this section, the outage performance of coordinated direct AF/DF relay assisted downlink NOMA is analyzed. We derive closed-form expressions of NU and FU outage probabilities considering $\mathrm{AF}$ or $\mathrm{DF}$ relaying. In order to determine the diversity order for $U_{N}$ and $U_{F}$, the asymptotic expressions of user outage probabilities are also derived. For ease of exposition, we define $\left|h_{B R}\right|^{2}=X\left(\lambda_{B R}=\lambda_{x}\right),\left|h_{R F}\right|^{2}=$ $Y\left(\lambda_{R F}=\lambda_{y}\right),\left|h_{B N}\right|^{2}=Z\left(\lambda_{B N}=\lambda_{z}\right),\left|h_{B F}\right|^{2}=W\left(\lambda_{B F}=\lambda_{w}\right)$, and $\left|h_{R N}\right|^{2}=V\left(\lambda_{R N}=\lambda_{v}\right)$. Note that the RVs X, Y, Z, W, $\mathrm{V}$ are independent and exponentially distributed with PDF $f_{\Omega}(\omega)=\lambda_{\omega} e^{-\lambda_{\omega} \omega}$ with $\Omega \in\{X, Y, Z, W, V\}, \omega \in\{x, y, z, w, v\}$.

\section{A. AF Relaying}

1) FU outage probability: The outage probability of $U_{F}$ can be written as $p_{F}^{A F}=\operatorname{Pr}\left\{\Gamma_{F}^{C O M}<\gamma_{F}\right\}$, where $\Gamma_{F}^{C O M}=$ $\Gamma_{F}^{I}+\Gamma_{F}^{I I}$. Using (6) and (11), $p_{F}^{A F}$ can be expressed as

$$
\begin{aligned}
& p_{F}^{A F}=\operatorname{Pr}\left\{\Gamma_{F F}^{C O M}<\gamma_{F}\right\} \\
& =\operatorname{Pr}\left\{\frac{\left(1-\alpha_{B}\right) \rho_{B} W}{\alpha_{B} \rho_{B} W+1}+\frac{\rho_{B} \rho_{R}\left(1-\alpha_{B}\right) X Y}{\rho_{B} \rho_{R} \alpha_{B} X Y+\rho_{R} Y+\rho_{B} X+1}<\gamma_{F}\right\} \\
& =\operatorname{Pr}\left\{\rho _ { R } Y \left[\rho_{B} X\left(\phi+\alpha_{B} \rho_{B} W\left(1-\alpha_{B}+\phi\right)\right)\right.\right. \\
& \left.\left.\quad-\left(\gamma_{F}-\rho_{B} W \phi\right)\right]<\left(\gamma_{F}-\rho_{B} W \phi\right)\left(1+\rho_{B} X\right)\right\}
\end{aligned}
$$

wherein $\phi=1-\alpha_{B}\left(1+\gamma_{F}\right)$. The sign of $\left(\gamma_{F}-\rho_{B} W \phi\right)$ depends on the range of $\mathrm{W}$ and different values of $\phi$. Depending on $\left(\gamma_{F}-\rho_{B} W \phi\right) \gtrless 0$, the conditions for outage on ranges of $X, Y$ and $W$ with $\phi>0$ $\left(\alpha_{B}<\frac{1}{1+\gamma_{F}}\right), \alpha_{B}-1<\phi<0\left(\frac{1}{1+\gamma_{F}} \leq \alpha_{B}<\frac{2}{2+\gamma_{F}}\right)$ and $\phi<\alpha_{B}-1\left(\alpha_{B} \geq \frac{2}{2+\gamma_{F}}\right)$ are listed in Table II wherein $\zeta_{F_{1}}(x, w)=\frac{\left(\gamma_{F}-\rho_{B} w \phi\right)\left(1+\rho_{B} x\right)}{\rho_{R}\left(\rho_{B} x\left(\phi+\alpha_{B} \rho_{B} w\left(1-\alpha_{B}+\phi\right)-\left(\gamma_{F}-\rho_{B} w \phi\right)\right)\right)}$,

\begin{tabular}{|c|c|c|}
\hline$\alpha_{B}<\frac{1}{1+\gamma_{F}}$ & $W<\chi_{F}$ & $\begin{array}{c}X<\zeta_{F_{1}}(W), Y>0 \\
X \geq \zeta_{F_{1}}(W), Y<\zeta_{F_{1}}(X, W)\end{array}$ \\
\hline \multirow{3}{*}{$\frac{1}{1+\gamma_{F}} \leq \alpha_{B}<\frac{2}{2+\gamma_{F}}$} & $W<\xi_{1}$ & $X>0, Y>0$ \\
\hline & \multirow[t]{2}{*}{$W \geq \xi_{1}$} & $X<\zeta_{F_{1}}(W), Y>0$ \\
\hline & & $X \geq \zeta_{F_{1}}(W), Y<\zeta_{F_{1}}(X, W)$ \\
\hline
\end{tabular}
$\zeta_{F_{1}}(w)=\frac{\left(\gamma_{F}-\rho_{B} w \phi\right)}{\rho_{B}\left(\phi+\alpha_{B} \rho_{B} w\left(1-\alpha_{B}+\phi\right)\right)}, \quad \chi_{F}=\frac{\gamma_{F}}{\phi \rho_{B}} \quad$ and
TABLE II: $\mathrm{U}_{F}$ outage - ranges of $Y, X$ and $W$ for different values of $\alpha_{B}$

$\xi_{1}=\frac{-\phi}{\alpha_{B} \rho_{B}\left(1-\alpha_{B}+\phi\right)}$. Now, $p_{F}^{A F}$ can be expressed as in (20) After some mathematical rearrangements (20) can be expressed as 21]. We first solve for $I_{1}$ as follows:

$I_{1}=\int_{o}^{\chi_{F}} \int_{\zeta_{1}(w)}^{\infty} \exp \left(-\frac{\lambda_{y} \zeta_{F_{1}}(w)\left(1+\rho_{B} x\right)}{\rho_{R}\left(x-\zeta_{F_{1}}(w)\right)}\right) \lambda_{x} e^{-\lambda_{x} x} d x \lambda_{w} e^{-\lambda_{w} w} d w$

Substituting $x=x-\zeta_{F_{1}}(w)$ in the above and re-arranging terms, we get

$$
\begin{aligned}
I_{1} & =\int_{o}^{\chi_{F}} \exp \left(-\zeta_{F_{1}}(w)\left(\lambda_{x}+\frac{\lambda_{y} \rho_{B}}{\rho_{R}}\right)\right) \int_{0}^{\infty} \lambda_{x} \\
& \times \exp \left(-\lambda_{x} x-\frac{\lambda_{y} \zeta_{F_{1}}(w)\left[1+\rho_{B} \zeta_{F_{1}}(w)\right]}{x \rho_{R}}\right) \lambda_{w} e^{-\lambda_{w} w} d x d w .
\end{aligned}
$$

Solving for the inner integral using [34, 3.324.1], we obtain

$$
\begin{gathered}
I_{1}=\int_{0}^{\chi_{F}} \exp \left(-\zeta_{F_{1}}(w)\left(\lambda_{x}+\frac{\lambda_{y} \rho_{B}}{\rho_{R}}\right)\right) 2 \sqrt{\mu_{F 1}(w)} \\
\times K_{1}\left(2 \sqrt{\mu_{F 1}(w)}\right) \lambda_{w} e^{-\lambda_{w} w} d w,
\end{gathered}
$$

where $\mu_{F_{1}}(w)=\frac{\lambda_{x} \lambda_{y} \zeta_{F_{1}}(w)\left[\rho_{B} \zeta_{F_{1}}(w)+1\right]}{\rho_{R}}$. Following a similar approach, we derive an expression for $I_{2} \cdot p_{F}^{A F}$ can then be expressed as

$$
p_{F}^{A F}= \begin{cases}1-e^{-\lambda_{w} \chi_{F}}-\int_{0}^{\chi_{F}} \exp \left(-\zeta_{F_{1}}(w)\left(\lambda_{x}+\frac{\lambda_{y} \rho_{B}}{\rho_{R}}\right)\right) 2 \sqrt{\mu_{F 1}(w)} \\ \times K_{1}\left(2 \sqrt{\mu_{F 1}(w)}\right) \lambda_{w} e^{-\lambda_{w} w} d w & \text { if } \alpha_{B}<\frac{1}{1+\gamma_{F}} \\ 1-\int_{\xi_{1}}^{\infty} \exp \left(-\zeta_{F_{1}}(w)\left(\lambda_{x}+\frac{\lambda_{y} \rho_{B}}{\rho_{R}}\right)\right) 2 \sqrt{\mu_{F 1}(w)} \\ K_{1}\left(2 \sqrt{\mu_{F 1}(w)}\right) \lambda_{w} e^{-\lambda_{w} w} d w & \text { if } \frac{1}{1+\gamma_{F}} \leq \alpha_{B}<\frac{2}{2+\gamma_{F}} \\ 1, & \text { otherwise. }\end{cases}
$$

Unfortunately, the integrals in 25] do not admit a closedform. We, therefore, present a highly accurate approximate expression for $P_{F}^{A F}$ in the following Theorem.

Theorem 1. An accurate closed-form expression for $F U$ 


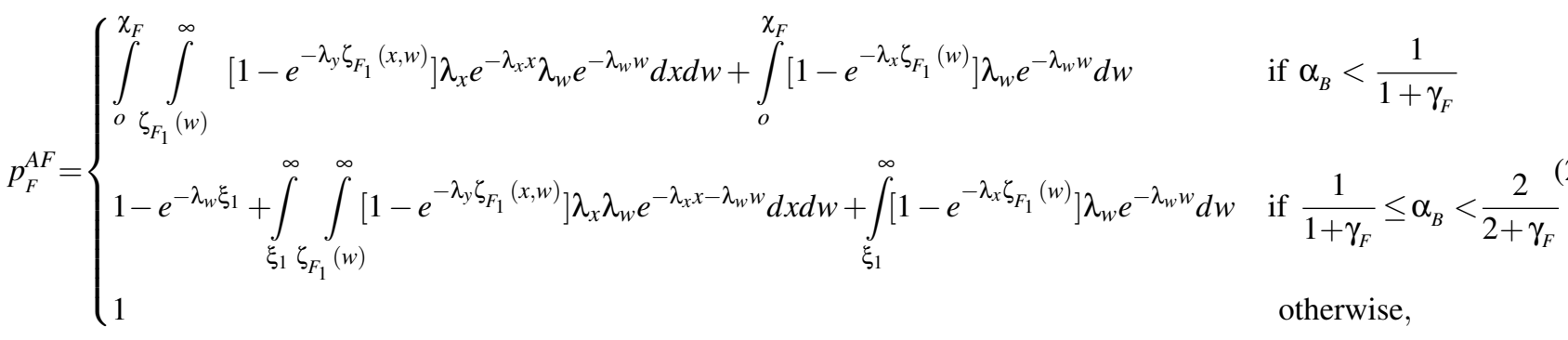

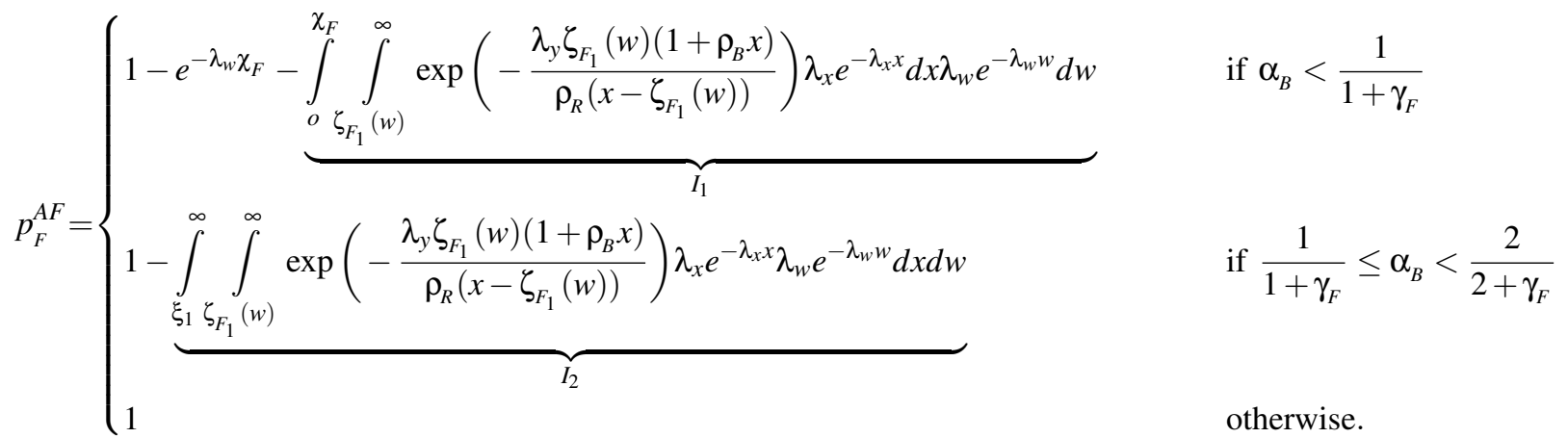

outage probability with $A F$ relaying is given by

$$
p_{F}^{A F}= \begin{cases}1-e^{-\lambda_{w} \chi_{F}}-e^{-\xi_{1}\left(\lambda_{w}+\frac{1}{\rho_{R}}\right)}\left[\Gamma\left(1,-\lambda_{w} \xi_{1} ; \vartheta_{6}\right)\right. \\ \left.-\Gamma\left(1, \lambda_{w}\left(\chi_{F}-\xi_{1}\right) ; \vartheta_{6}\right)\right] \quad \text { if } \alpha_{B}<\frac{1}{1+\gamma_{F}} \\ 1-e^{-\xi_{1}\left(\lambda_{w}+\frac{1}{\rho_{R}}\right)} \sqrt{4 \vartheta_{6}} K_{1}\left(\sqrt{4 \vartheta_{6}}\right) & \text { if } \frac{1}{1+\gamma_{F}} \leq \alpha_{B}<\frac{2}{2+\gamma_{F}} \\ 1 & \text { otherwise. }\end{cases}
$$

Proof. Refer to Appendix A.

Using the expression obtained in 26, it is extremely difficult to establish the diversity order. We, therefore, derive a high-SNR approximation to $p_{F}^{A F}$ in the following Lemma.

Lemma 1. A high-SNR approximation to $p_{F}^{A F}$ is given by

$$
p_{F}^{A F} \simeq\left\{\begin{array}{l}
\left(\lambda_{x}+\frac{\lambda_{y} \rho_{B}}{\rho_{R}}\right) \xi_{1}\left[1-e^{-\lambda_{w} \chi_{F}}+\left(\xi_{1}-\chi_{F}\right) \lambda_{w} e^{-\lambda_{w} \xi_{1}}\right. \\
\left.\left[\mathcal{E}_{1}\left(-\lambda_{w} \xi_{1}\right)-\mathcal{E}_{1}\left(-\lambda_{w} \xi_{1}+\lambda_{w} \chi_{F}\right)\right]\right] \text { if } \alpha_{B}<\frac{1}{1+\gamma_{F}} \\
1-\frac{e^{-\lambda_{w} \xi_{1}}}{1+\xi_{1}\left(\lambda_{x}+\lambda_{y}\right)} \quad \text { if } \frac{1}{1+\gamma_{F}} \leq \alpha_{B}<\frac{2}{2+\gamma_{F}} \\
\text { otherwise. }
\end{array}\right.
$$

Proof. Refer to Appendix B.

Remark 1. In the absence of direct link to $U_{F}$, the outage probability of $U_{F}$ can be readily derived by substituting $W=$ 0 into (19). After using a procedure similar to that used to derive (25), we obtain an exact closed-form expression for
$p_{F}^{A F}$ as follows:

$$
\begin{aligned}
p_{F}^{A F} & =1-\exp \left(-\frac{\chi_{F}}{\rho_{R}}\left(\lambda_{x} \rho_{R}+\lambda_{y} \rho_{B}\right)\right) \\
& \times 2 \sqrt{\mu_{F}} K_{1}\left(2 \sqrt{\mu_{F}}\right), \quad \text { if } \alpha_{B}<\frac{1}{1+\gamma_{F}}
\end{aligned}
$$

where $\mu_{F}=\frac{\lambda_{x} \lambda_{y} \chi_{F}\left(1+\rho_{B} \chi_{F}\right)}{\rho_{R}}$. The above equality holds only for $\alpha_{B}<\frac{1}{1+\gamma_{F}}$, otherwise $p_{F}^{A F}=1$. Note that for higher values of $\rho_{B}$ and $\rho_{R}, \mu_{F}$ and $\chi_{F}$ are very small. Therefore, applying $K_{1}(\theta) \simeq \frac{1}{2} \Gamma(1)\left(\frac{\theta}{2}\right)^{-1}$ [35. 9.6.9] in [28], we obtain

$$
p_{F}^{A F} \simeq 1-\exp \left(-\chi_{F}\left[\lambda_{x}+\frac{\lambda_{y} \rho_{B}}{\rho_{R}}\right]\right) .
$$

Using $e^{-\theta} \simeq 1-\theta$ in the above, a high-SNR approximation to $p_{F}^{A F}$ is given by

$$
p_{F}^{A F} \simeq \frac{\gamma_{F}\left[\rho_{R} \lambda_{x}+\rho_{B} \lambda_{y}\right]}{\rho_{R} \rho_{B} \phi} .
$$

2) NU Outage Probability: Let $\Gamma_{N F}^{C O M}=\Gamma_{N F}^{I}+\Gamma_{N F}^{I I}$ and $\Gamma_{N N}^{C O M}=\Gamma_{N N}^{I}+\Gamma_{N N}^{I I}$. Due to the manner in which SIC is performed with combining, the outage probability of $\mathrm{U}_{N}$ is

$$
p_{N}^{A F}=1-\operatorname{Pr}\left\{\Gamma_{N F}^{C O M} \geq \gamma_{F}, \Gamma_{N N}^{C O M} \geq \gamma_{N}\right\} .
$$

Theorem 2. The NU outage probability can be written as in 32, where $\chi_{N}=\frac{\gamma_{N}}{\alpha_{B} \rho_{B}}, \quad \chi_{N_{1}}(Z)=\frac{\gamma_{N}-\alpha_{B} Z \rho_{B}}{\rho_{B} \alpha_{B}}$, $\zeta_{N_{1}}(X, Z)=\frac{\chi_{N_{1}}(Z)\left(\rho_{B} X+1\right)}{\rho_{R}\left(X-\chi_{N_{1}}(Z)\right)}, \quad \chi_{N_{2}}(Z)=\frac{\gamma_{F}-\phi Z \rho_{B}}{\rho_{B}\left[\phi+\alpha_{B} Z \rho_{B}\left(1-\alpha_{B}+\phi\right)\right]}$ and $\zeta_{N_{2}}(X, Z)=\frac{\chi_{N_{2}}(Z)\left(\rho_{B} X+1\right)}{\rho_{R}\left(X-\chi_{N_{2}}(Z)\right)}$. 


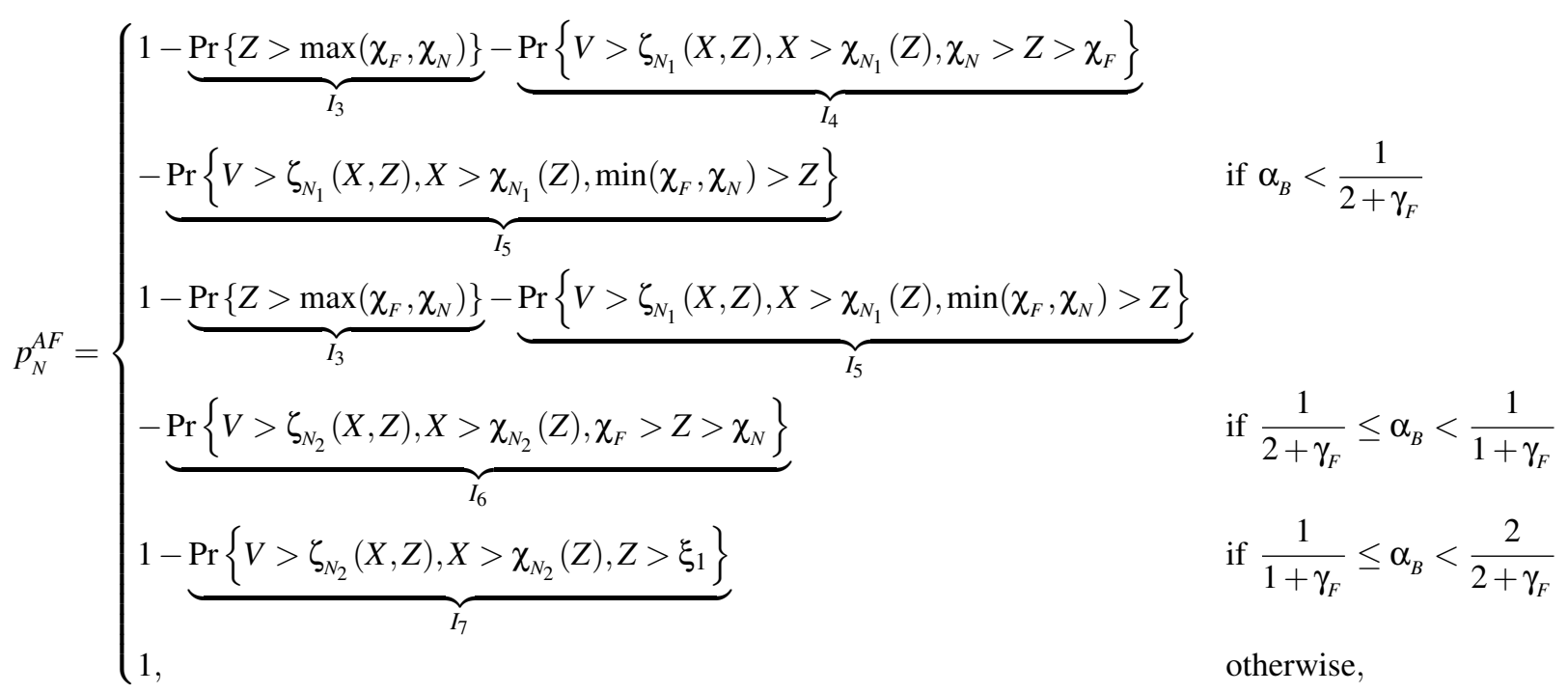

Proof. Using (4), (5), 9) and (10) into (31), we obtain

$$
\begin{aligned}
& p_{N}^{A F}=1-\operatorname{Pr}\left\{\frac{\left(1-\alpha_{B}\right) \rho_{B} Z}{\alpha_{B} \rho_{B} Z+1}+\frac{\rho_{B} \rho_{R}\left(1-\alpha_{B}\right) X V}{\rho_{B} \rho_{R} \alpha_{B} X V+\rho_{R} V+\rho_{B} X+1}\right. \\
&\left.\geq \gamma_{F}, \alpha_{B} \rho_{B} Z+\frac{\rho_{B} \rho_{R} \alpha_{B} X V}{\rho_{R} V+\rho_{B} X+1} \geq \gamma_{N}\right\} \\
&=1-\operatorname{Pr}\left\{\left[V \rho _ { R } \left(X \rho_{B}\left(\phi+\alpha_{B} \rho_{B} Z\left(1-\alpha_{B}+\phi\right)\right)-\left(\gamma_{F}\right.\right.\right.\right. \\
&\left.\left.\left.-\phi \rho_{B} Z\right)\right) \geq\left(\gamma_{F}-\phi \rho_{B} Z\right)\left(1+\rho_{B} X\right)\right],\left[V \rho _ { R } \left(X \rho_{B} \alpha_{B}\right.\right. \\
&\left.\left.\left.-\left(\gamma_{N}-\alpha_{B} \rho_{B} Z\right)\right) \geq\left(\gamma_{N}-\alpha_{B} \rho_{B} Z\right)\left(1+\rho_{B} X\right)\right]\right\} .
\end{aligned}
$$

In the above, depending on values of $\alpha_{B}$ and $\gamma_{F}$, two ranges of $\alpha_{B}$ exist i.e. $\alpha_{B}<\frac{1}{1+\gamma_{F}}$ or $\alpha_{B} \geq \frac{1}{1+\gamma_{F}}$. Note that the signs of $\left(\gamma_{F}-\phi \rho_{B} Z\right)$ and $\left(\gamma_{N}-\alpha_{B} \rho_{B} Z\right)$ depend on the range of $Z$. Based on the ranges of RVs $V, X$ and $Z$, the conditions for non-outage probability of $U_{N}$ for different values of $\alpha_{B}$ are listed in Table III Now, $p_{N}^{A F}$ can be expressed as

$p_{N}^{A F}=1-\operatorname{Pr}\left\{V>\zeta_{N_{2}}(X, Z), X>\chi_{N_{2}}(Z), Z>\xi_{1}\right\} U(-\phi)$

$-\left[\operatorname{Pr}\left\{Z>\max \left(\chi_{N}, \chi_{F}\right)\right\}+\operatorname{Pr}\left\{V>\zeta_{N_{1}}(X, Z), X>\chi_{N_{1}}(Z)\right.\right.$,

$\left.\left.\chi_{N}>Z>\chi_{F}\right\} U\left(\chi_{N}-\chi_{F}\right\}\right)+\operatorname{Pr}\left\{V>\zeta_{N_{2}}(X, Z), X>\chi_{N_{2}}(Z)\right.$,

$\left.\chi_{F}>Z>\chi_{N}\right\} U\left(\chi_{F}-\chi_{N}\right)+\operatorname{Pr}\left\{V>\zeta_{N_{1}}(X, Z), X>\chi_{N_{1}}(Z)\right.$,

$\left.\left.Z<\min \left(\chi_{N}, \chi_{F}\right)\right\}\right] U(\phi)$,

where $\chi_{N_{1}}(Z), \chi_{N_{2}}(Z), \zeta_{N_{1}}(X, Z), \zeta_{N_{2}}(X, Z), \chi_{F}, \chi_{N}$ and $\phi$ are defined in the line following (32). After performing mathematical rearrangements in (34) we obtain 32).

Theorem 3. An accurate closed-form expression for $p_{N}^{A F}$ is given by (35), where $\vartheta_{2}=\xi_{1}\left(\xi_{1}+\frac{\gamma_{F}}{\rho_{B} \phi}\right) C_{\ell}, C=C_{\ell}-\lambda_{z}$ and $C_{\ell}=\lambda_{x}+\frac{\lambda_{v} \rho_{B}}{\rho_{R}}$.

Proof. Refer to Appendix C.

Lemma 2. The high-SNR expression for $p_{N}^{A F}$ is given by

Proof. Refer to Appendix C.
Lemma 3. The diversity orders $D_{F}^{A F}$ and $D_{N}^{A F}$ achieved at $U_{F}$ and $U_{N}$ are given by

$$
\begin{aligned}
& D_{F}^{A F}=\left\{\begin{array}{ll}
2, & \text { if } \alpha_{B}<\frac{1}{1+\gamma_{F}} \\
1, & \text { if } \frac{1}{1+\gamma_{F}} \leq \alpha_{B}<\frac{2}{2+\gamma_{F}} \\
0, & \text { otherwise. }
\end{array},\right. \\
& D_{N}^{A F}= \begin{cases}2, & \text { if } \alpha_{B}<\frac{1}{1+\gamma_{F}} \\
1, & \text { if } \frac{1}{1+\gamma_{F}} \leq \alpha_{B}<\frac{2}{2+\gamma_{F}} \\
0, & \text { otherwise. }\end{cases}
\end{aligned}
$$

Proof. To derive the diversity order for $\mathrm{U}_{F}$ and $\mathrm{U}_{N}$, we use the high-SNR expression of $p_{F}^{A F}$ and $p_{N}^{A F}$ given by 27) and (35), respectively. We consider $\rho_{B}=\rho_{R}=\rho$ without loss of generality. The diversity orders attained by $\mathrm{U}_{F}$ and $\mathrm{U}_{N}$ are given by $D_{j}^{A F}=-\lim _{\rho \rightarrow \infty} \frac{\log _{2} p_{j}^{A F}(\rho)}{\log _{2} \rho}, j \in\{N, F\}$. For $\rho \rightarrow$ $\infty$, we use $e^{-\theta}=1-\theta$ and substitute for $\chi_{F}$ and $\xi_{1}$. After neglecting higher order terms of $\frac{1}{\rho}$, we obtain diversity order as expressed in (37). The detailed proof is omitted due to paucity of space.

Remark 2. The NU also attains a diversity of two is significant and motivates it to participate in NOMA signalling.

Remark 3. In the absence of direct link to $U_{F}$, the diversity order $D_{F}^{A F}$ is obtained by using $\rho_{B}=\rho_{R}=\rho$ into 30 . The diversity order $D_{F}^{A F}$ can be expressed as

$$
D_{F}^{A F}= \begin{cases}1, & \text { if } \alpha_{B}<\frac{1}{1+\gamma_{F}} \\ 0, & \text { otherwise. }\end{cases}
$$

However, the diversity order for $U_{N}$ remains the same as expressed in 37. 
TABLE III: $\mathrm{U}_{N}$ non-outage - ranges of $Z, X$ and $V$ for different values of $\alpha_{B}$

\begin{tabular}{|c|c|c|}
\hline \multirow{4}{*}{$\alpha_{B}<\frac{1}{1+\gamma_{F}}$} & $Z>\max \left(\chi_{F}, \chi_{N}\right)$ & $V>0, X>0$ \\
\hline & $\chi_{N}>Z>\chi_{F}$ & $V>\zeta_{N_{1}}(X, Z), X>\chi_{N_{1}}(Z)$ \\
\hline & $\chi_{F}>Z>\chi_{N}$ & $V>\zeta_{N_{2}}(X, Z), X>\chi_{N_{2}}(Z)$ \\
\hline & $\min \left(\chi_{F}, \chi_{N}\right)>Z$ & $\begin{array}{r}V>\max \left(\zeta_{N_{1}}(X, Z), \zeta_{N_{2}}(X, Z)\right), X>\max \left(\chi_{N_{1}}(Z), \chi_{N_{2}}(Z)\right) \\
\text { if } \chi_{N_{1}}(Z)>\chi_{N_{2}}(Z) \Rightarrow \zeta_{N_{1}}(X, Z)>\zeta_{N_{2}}(X, Z) \Rightarrow Z<\chi_{N} \\
\text { if } \chi_{N_{1}}(Z)>\chi_{N_{2}}(Z) \Rightarrow \zeta_{N_{1}}(X, Z)<\zeta_{N_{2}}(X, Z) \text { not valid }\end{array}$ \\
\hline$\frac{1}{1+\gamma_{F}} \leq \alpha_{B}<\frac{2}{2+\gamma_{F}}$ & $Z>\xi_{1}$ & $V>\zeta_{N_{2}}(X, Z), X>\chi_{N_{2}}(Z)$ \\
\hline$\alpha_{B} \geq \frac{2}{2+\gamma_{F}}$ & & Outage always occurs \\
\hline
\end{tabular}

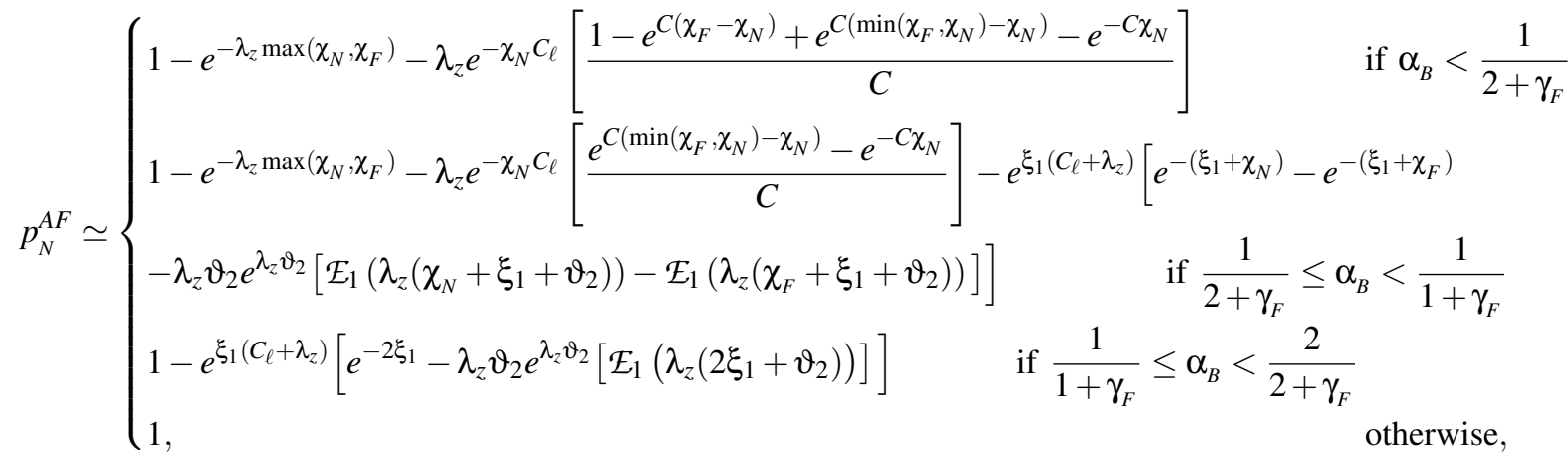

$$
p_{N}^{A F} \approx \begin{cases}1-e^{-\lambda_{z} \max \left(\chi_{N}, \chi_{F}\right)}-\frac{\lambda_{z} e^{-\chi_{N} C_{\ell}}\left[1-e^{-C \chi_{N}}\left(1+e^{C \chi_{F}}-e^{C \min \left(\chi_{F}, \chi_{N}\right)}\right)\right]}{C} & \text { if } \alpha_{B}<\frac{1}{2+\gamma_{F}} \\ 1-e^{-\lambda_{z} \max \left(\chi_{N}, \chi_{F}\right)}-\frac{\lambda_{z} e^{-2 \chi_{N} C_{\ell}}\left(e^{C \min \left(\chi_{F}, \chi_{N}\right)}-1\right)}{C}+\frac{\lambda_{z} \vartheta_{2} e^{-\lambda_{z} \xi_{1}}\left(e^{-\lambda_{z} \chi_{N}}-e^{\left.-\lambda_{z} \chi_{F}\right)}\right.}{1+\lambda_{z}\left(\chi_{N}+\xi_{1}+\vartheta_{2}\right)} \\ -e^{\xi_{1}\left(C_{\ell}+\lambda_{z}-1\right)}\left(e^{\left.-\chi_{N}-e^{-\chi_{F}}\right)}\right. & \text { if } \frac{1}{2+\gamma_{F}} \leq \alpha_{B}<\frac{1}{1+\gamma_{F}} \\ 1-e^{\xi_{1}\left(\lambda_{z}-C_{\ell}\right)}+\frac{\lambda_{z} \vartheta_{2} e^{\xi_{1}\left(C_{\ell}-\lambda_{z}\right)}}{1+\lambda_{z}\left(\vartheta_{2}+2 \xi_{1}\right)} & \text { if } \frac{1}{1+\gamma_{F}} \leq \alpha_{B}<\frac{2}{2+\gamma_{F}} \\ 1, & \text { otherwise }\end{cases}
$$

\section{B. DF Relaying}

1) $F U$ outage probability: Let $\Gamma_{F F-S}^{C O M}=\Gamma_{F F}^{I}+\Gamma_{F F-S}^{I I}$ and $\Gamma_{F F-F}^{C O M}=\Gamma_{F F}^{I}+\Gamma_{F F-F}^{I I}$. FU is not in outage when a) both $s_{F}$ and $s_{N}$ are decoded at $\mathrm{R}\left(\Gamma_{R F}^{I} \geq \gamma_{F}, \Gamma_{R N}^{I} \geq \gamma_{N}\right)$ and $\mathrm{FU}$ SNR after combining is sufficient $\left(\Gamma_{F F-S}^{C O M} \geq \gamma_{F}\right)$, b) $s_{F}$ can be decoded at R, but not $s_{N}$ but SNR after combining is sufficient at $\mathrm{FU}\left(\Gamma_{F F-F}^{C O M} \geq \gamma_{F}\right)$, and c) $s_{F}$ cannot be decoded at $\mathrm{R}$ but $\Gamma_{F F}^{I} \geq \gamma_{F}$. The outage probability for $\mathrm{U}_{\mathrm{F}}$ can be formulated as

$$
\begin{aligned}
p_{F}^{D F}=1 & -\operatorname{Pr}\{\underbrace{\Gamma_{R F}^{I} \geq \gamma_{F}, \Gamma_{R N}^{I} \geq \gamma_{N}, \Gamma_{F F-S}^{C O M} \geq \gamma_{F}}_{A_{1}}\} \\
& -\operatorname{Pr}\{\underbrace{\Gamma_{R F}^{I} \geq \gamma_{F}, \Gamma_{R N}^{I}<\gamma_{N}, \Gamma_{F F-F}^{C O M} \geq \gamma_{F}}_{A_{2}}\} \\
& -\operatorname{Pr}\{\underbrace{\Gamma_{R F}^{I}<\gamma_{F}, \Gamma_{F F}^{I} \geq \gamma_{F}}_{A_{3}}\},
\end{aligned}
$$

In the following, we present closed-form expressions for FU outage.

Theorem 4. The exact closed-form expression of the outage probability for $U_{F}$ is given by where $\phi_{R}=1-\alpha_{R}\left(1+\gamma_{F}\right)$, $\vartheta_{1}=\rho_{B}\left(\alpha_{B}\left(1-\alpha_{R}\right)+\alpha_{R} \phi\right), \varsigma_{1}=\frac{\lambda_{w} \phi_{R}}{\vartheta_{1}}$ and $\varsigma_{2}=\chi_{F}+\frac{\phi_{R}}{\vartheta_{1}}$

Proof. Refer to Appendix D

The expressions obtained in 40 involve the generalized incomplete gamma function due to which the diversity cannot readily be established from them. We, therefore, present a highly accurate high-SNR approximation to $p_{N}^{A F}$ in the following Lemma.

Lemma 4. A high-SNR approximation to $p_{F}^{D F}$ is given by (41).

Proof. Refer to Appendix E]

Remark 4. In the absence of the direct link to $U_{F}$, we can use $W=0$ in (39) to get 


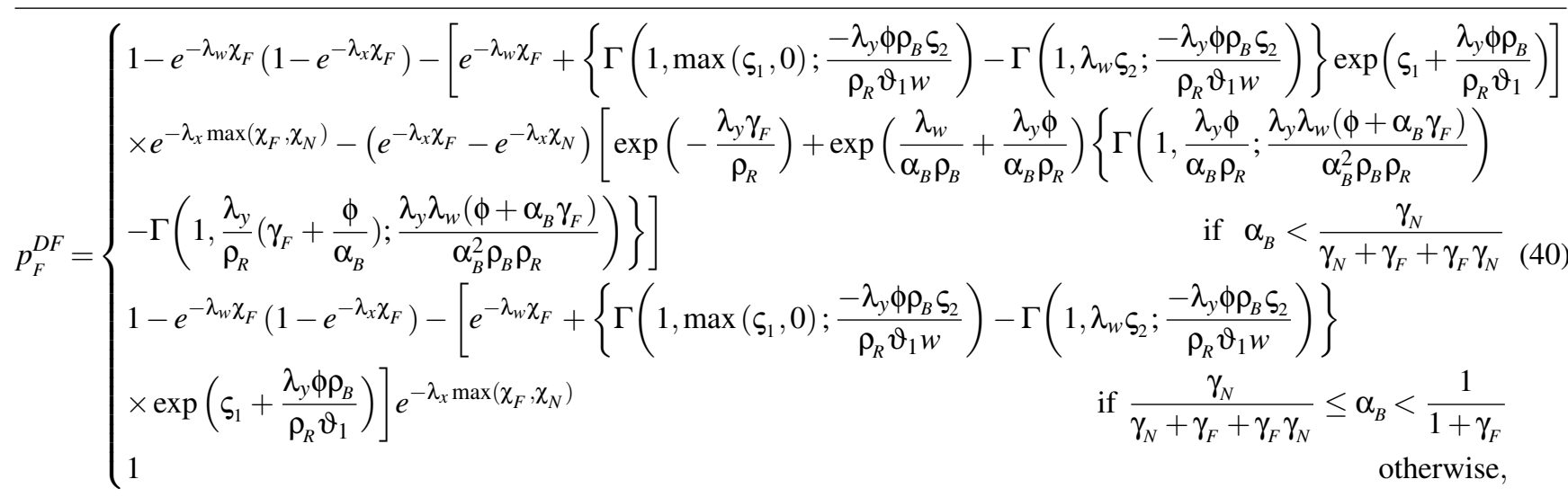

$$
p_{F}^{D F} \simeq\left\{\begin{array}{rr}
1+e^{-\lambda_{w} \chi_{F}}\left(1-e^{-\lambda_{x} \chi_{F}}\right)-e^{-\lambda_{x} \chi_{N}}\left[\exp \left(\varsigma_{1}+\frac{\lambda_{y} \phi \rho_{B}}{\rho_{R} \vartheta_{1}}-\max \left(\varsigma_{1}, 0\right)\right)+e^{-\lambda_{w} \chi_{F}}\left(1-\exp \left(\frac{\lambda_{y} \phi \rho_{B}}{\rho_{R} \vartheta_{1}}\right)\right)\right] \\
-\left(e^{-\lambda_{x} \chi_{F}}-e^{-\lambda_{x} \chi_{N}}\right)\left[\exp \left(\frac{\lambda_{w}}{\alpha_{B} \rho_{B}}\right)+\exp \left(-\frac{\lambda_{y} \gamma_{F}}{\rho_{R}}\right)\left(1-\exp \left(\frac{\lambda_{w}}{\alpha_{B} \rho_{B}}\right)\right)\right] & \text { if } \alpha_{B}<\frac{\gamma_{N}}{\gamma_{N}+\gamma_{F}+\gamma_{F} \gamma_{N}} \\
1+e^{-\lambda_{w} \chi_{F}}\left(1-e^{-\lambda_{x} \chi_{F}}\right)-e^{-\lambda_{x} \chi_{F}}\left[\exp \left(\varsigma_{1}+\frac{\lambda_{y} \phi \rho_{B}}{\rho_{R} \vartheta_{1}}-\max \left(\varsigma_{1}, 0\right)\right)\right. & \text { if } \frac{\gamma_{N}}{\gamma_{N}+\gamma_{F}+\gamma_{F} \gamma_{N}} \leq \alpha_{B}<\frac{1}{1+\gamma_{F}} \\
\left.+e^{-\lambda_{w} \chi_{F}}\left(1-\exp \left(\frac{\lambda_{y} \phi \rho_{B}}{\rho_{R} \vartheta_{1}}\right)\right)\right] & \text { otherwise. }
\end{array}\right.
$$

$$
\begin{gathered}
p_{F}^{D F}=1-\operatorname{Pr}\left\{X>\max \left(\chi_{N}, \chi_{F}\right), Y \phi_{R} \rho_{R}>\gamma_{F}\right\} \\
-\operatorname{Pr}\left\{\chi_{F}<X<\chi_{N}, Y \rho_{R}>\gamma_{F}\right\} .
\end{gathered}
$$

After solving (42) as in Theorem 4 an exact expression for $p_{F}^{D F}$ is given by (43). Using $e^{-\vartheta} \simeq 1-\theta$ in above and neglecting higher order terms of $\frac{1}{\rho_{R}}$, a high-SNR approximation to $p_{F}^{D F}$ is given by

$$
p_{F}^{D F} \simeq \begin{cases}\frac{\lambda_{y} \gamma_{F}}{\rho_{R} \phi_{R}}+\lambda_{x} \chi_{F} & \text { if } \alpha_{B}<\frac{1}{1+\gamma_{F}} \\ 1 & \text { otherwise. }\end{cases}
$$

2) NU outage probability: NU is not in outage when a) $\mathrm{R}$ decodes both $s_{F}$ and $s_{N}\left(\Gamma_{R F}^{I} \geq \gamma_{F}, \Gamma_{R N}^{I} \geq \gamma_{N}\right)$ and SNRs at NU (after combining) are sufficient to decode both $s_{F}$ and $s_{N}\left(\Gamma_{N F-S}^{C O M} \geq \gamma_{F}, \Gamma_{N N-S}^{C O M} \geq \gamma_{N}\right)$, b) R decodes $s_{F}$ but fails to decode $s_{N}\left(\Gamma_{R F}^{I} \geq \gamma_{F}, \Gamma_{R N}^{I}<\gamma_{N}\right)$, and SNRs to decode $s_{F}$ (after combining) and $s_{N}$ (from first phase) are sufficient at $\mathrm{NU}\left(\Gamma_{N F-F}^{C O M} \geq \gamma_{F}, \Gamma_{N N}^{I} \geq \gamma_{N}\right)$, and c) R fails to decode $s_{F}$ $\left(\Gamma_{R F}^{I}<\gamma_{F}\right)$, but the first phase SNRs are sufficient to decode both $s_{F}$ and $s_{N}$ at $\mathrm{NU}\left(\Gamma_{N F}^{I} \geq \gamma_{F}, \Gamma_{N N}^{I} \geq \gamma_{N}\right)$. The outage probability for $\mathrm{U}_{\mathrm{N}}$ can be formulated as

$$
\begin{aligned}
p_{N}^{D F} & =1-\operatorname{Pr}\{\underbrace{\Gamma_{R F}^{I}<\gamma_{F}, \Gamma_{N F}^{I} \geq \gamma_{F}, \Gamma_{N N}^{I} \geq \gamma_{N}}_{B_{1}}\} \\
& -\operatorname{Pr}\{\underbrace{\Gamma_{R F}^{I} \geq \gamma_{F}, \Gamma_{R N}^{I}<\gamma_{N}, \Gamma_{N F-F}^{C O M} \geq \gamma_{F}, \Gamma_{N N}^{I} \geq \gamma_{N}}_{B_{2}}\} \\
& -\operatorname{Pr}\{\underbrace{\Gamma_{R F}^{I} \geq \gamma_{F}, \Gamma_{R N}^{I} \geq \gamma_{N}, \Gamma_{N F-S}^{C O M} \geq \gamma_{F}, \Gamma_{N N-S}^{C O M} \geq \gamma_{N}}_{B_{3}}\},
\end{aligned}
$$

where $\Gamma_{N F-F}^{C O M}=\Gamma_{N F}^{I}+\Gamma_{N F-F}^{I I}, \quad \Gamma_{N F-S}^{C O M}=\Gamma_{N F}^{I}+\Gamma_{N F-S}^{I I}$ and $\Gamma_{N N-S}^{C O M}=\Gamma_{N}^{I}+\Gamma_{N N-S}^{I I}$.
Theorem 5. The exact closed-form expression of the outage probability for $U_{N}$ is given by [46], where $\vartheta_{4}=$ $\frac{\phi \alpha_{R}+\gamma_{N} \phi \alpha_{R}+\gamma_{N} \alpha_{B}\left(1-\alpha_{R}\right)-\alpha_{B} \phi_{R}}{\rho_{B} \alpha_{B}\left(\phi \alpha_{R}+\alpha_{B}\left(1-\alpha_{R}\right)\right)}, \quad \vartheta_{5}=\frac{-\phi_{R}}{\vartheta_{1}}, \quad \vartheta_{1}=\rho_{B}\left(\phi \alpha_{R}+\right.$ $\left.\alpha_{B}\left(1-\alpha_{R}\right)\right), \tilde{\chi_{F}}=\frac{\lambda_{z} \lambda_{\nu} \phi \rho_{B}\left(\chi_{F}+\frac{\phi_{R}}{\vartheta_{1}}\right)}{\vartheta_{1}}$ and $\tilde{\chi_{N}}=\min \left(\chi_{F}, \chi_{N}, \vartheta_{4}\right)$.

Proof. Refer to Appendix F

In the following Lemma, we present a high-SNR approximate expression for $p_{N}^{D F}$ to obtain the diversity order at NU.

Lemma 5. A high-SNR approximation to $p_{N}^{D F}$ is given by 47), where $\varepsilon_{3}=\frac{\lambda_{v}}{\alpha_{R} \rho_{R}}$.

Proof. The proof is similar to that used to derive (41) and is therefore omitted.

Lemma 6. The diversity orders $D_{F}^{D F}$ and $D_{N}^{D F}$ attained by $U_{F}$ and $U_{N}$ can be expressed as

$$
\begin{aligned}
& D_{F}^{D F}=\left\{\begin{array}{ll}
2 & \text { if } \alpha_{B}<\frac{1}{1+\gamma_{F}}, \alpha_{R}<\frac{1}{1+\gamma_{F}} \\
1 & \text { if } \alpha_{B}<\frac{1}{1+\gamma_{F}}, \alpha_{R} \geq \frac{1}{1+\gamma_{F}} \\
0 & \text { otherwise. }
\end{array},\right. \\
& D_{N}^{D F}= \begin{cases}2 & \text { if } \alpha_{B}<\frac{1}{1+\gamma_{F}}, \alpha_{R}<\frac{1}{1+\gamma_{F}} \\
1 & \text { if } \alpha_{B}<\frac{1}{1+\gamma_{F}}, \alpha_{B} \geq \frac{1}{1+\gamma_{F}} \\
0 & \text { otherwise. }\end{cases}
\end{aligned}
$$

Proof. The diversity orders for $\mathrm{U}_{F}$ and $\mathrm{U}_{\mathrm{N}}$ are obtained by using high-SNR expressions of $p_{F}^{D F}$ and $p_{N}^{D F}$ given by (41) 


$$
p_{F}^{D F}= \begin{cases}1-\exp \left(-\frac{\lambda_{y} \gamma_{F}}{\rho_{R} \phi_{R}}-\lambda_{x} \chi_{N}\right)-\exp \left(-\frac{\lambda_{y} \gamma_{F}}{\rho_{R}}\right)\left[e^{-\lambda_{x} \chi_{F}}-e^{\left.-\lambda_{x} \chi_{N}\right]}\right. & \text { if } \alpha_{B}<\frac{\gamma_{N}}{\gamma_{N}+\gamma_{F}+\gamma_{F} \gamma_{N}} \\ 1-\exp \left(-\frac{\lambda_{y} \gamma_{F}}{\rho_{R} \phi_{R}}-\lambda_{x} \chi_{F}\right) & \text { if } \frac{\gamma_{N}}{\gamma_{N}+\gamma_{F}+\gamma_{F} \gamma_{N}} \leq \alpha_{B}<\frac{1}{1+\gamma_{F}} \\ 1 & \text { otherwise. }\end{cases}
$$

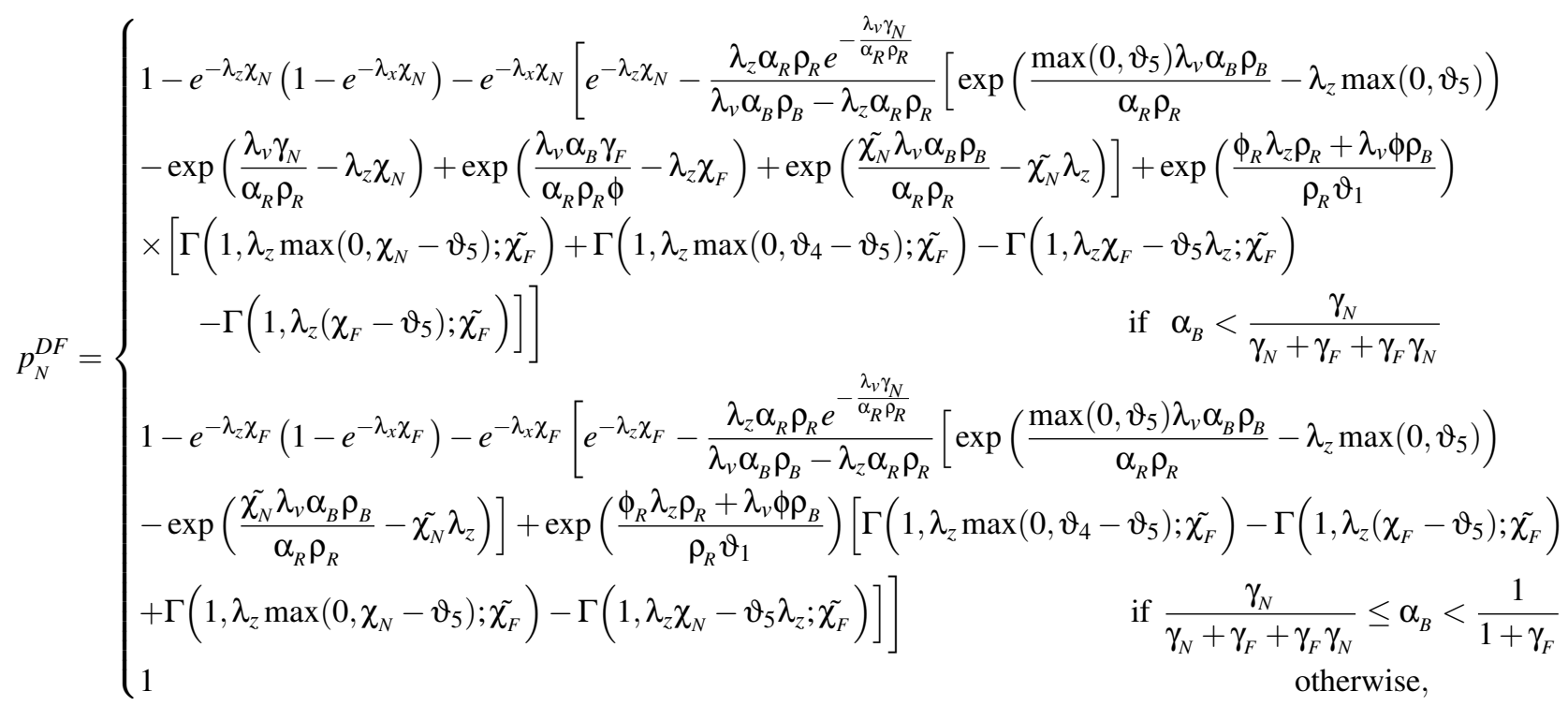

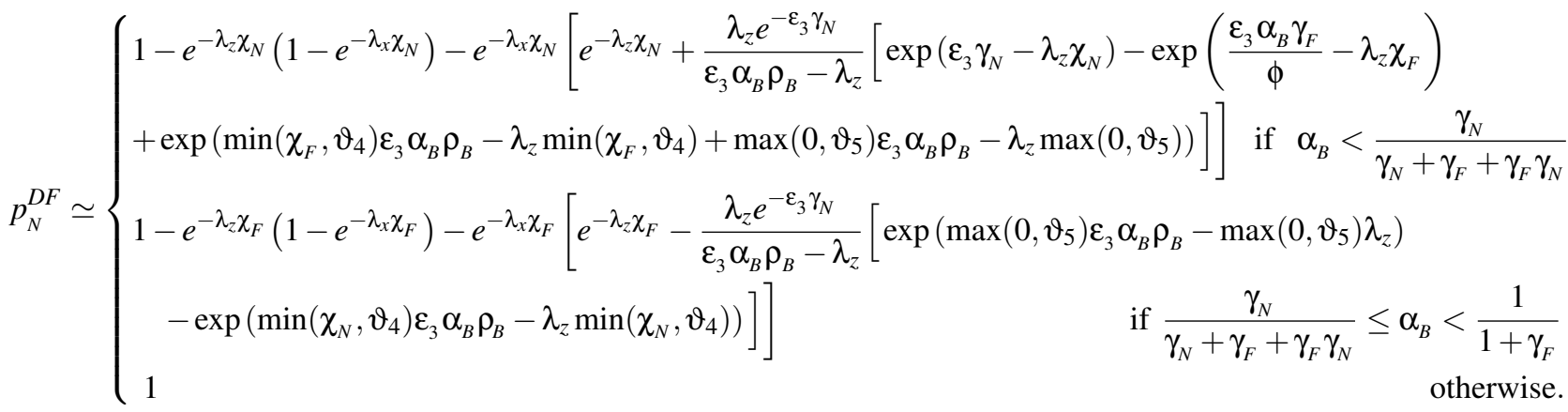

and (47), respectively. We substitute $\rho_{B}=\rho_{R}=\rho$ and solve for $D_{j}^{D F}=-\lim _{\rho \rightarrow \infty} \frac{\log _{2} p_{j}^{D F}(\rho)}{\log _{2} \rho}, j \in\{N, F\}$. Details are omitted due to paucity of space.

Remark 5. In the absence of direct link to $U_{F}$, the diversity order $D_{F}^{D F}$ is obtained by using $\rho_{B}=\rho_{R}=\rho \rightarrow \infty$ into 43. and using linear approximations to the exponential terms. The diversity order $D_{F}^{D F}$ can be expressed as

$$
D_{F}^{D F}= \begin{cases}1 & \text { if } \alpha_{B}<\frac{1}{1+\gamma_{F}}, \alpha_{R}<\frac{1}{1+\gamma_{F}} . \\ 0 & \text { otherwise. }\end{cases}
$$

However, the diversity order for $U_{N}$ remains the same as in (48.

\section{NU THROUGHPUT AND EE MAXIMIZATION}

NU needs to decode the FU symbol, perform SIC to decode its own symbol, and might therefore incur some perfor- mance loss. For this reason, maximizing the NU throughput while guaranteeing the desired FU target throughput $\tilde{\tau}$ is well motivated. We do so in this section. The FU and NU throughput in bits per channel use (bpcu) is defined as

$$
\tau_{j}^{i}=\frac{1}{2} R_{j}\left(1-p_{j}^{i}\right), \quad j \in\{N, F\}, i \in\{A F, D F\}
$$

where $p_{F}^{A F}, p_{N}^{A F}, p_{F}^{D F}$ and $p_{N}^{D F}$ are as in 26, (35), 40, and (46) respectively.

Careful choice of power allocation coefficient $\alpha_{B}$ and symbol rates $R_{F}$ and $R_{N}$ is crucial to attaining good throughput at $\mathrm{U}_{\mathrm{N}}$ and $\mathrm{U}_{\mathrm{F}}$. With DF relays, $\alpha_{R}$ can also be chosen. However, it will be demonstrated in Section $\mathrm{V}$ that a large range of $\alpha_{R}$ values result in the same throughput performance. The reason for this is that $\alpha_{R}$ is only used when both $s_{F}$ and $s_{N}$ are decoded correctly at R (which in turn depends on $\alpha_{B}$ ). We, therefore, focus only on the choice of $\alpha_{B}, R_{N}$, and $R_{F}$.

We first consider the case when the $\mathrm{B}-\mathrm{U}_{\mathrm{F}}$ link is absent. 
Noting that the NU benefits when the maximum value of $\alpha_{B}$ is chosen, we derive $\alpha_{B}^{i^{o p t}}$ by using (30) and 440 into (50) to get

$$
\begin{aligned}
& \alpha_{B}^{A F^{o p t}} \simeq \frac{1}{1+\gamma_{F}}-\frac{\gamma_{F} R_{F}\left(\lambda_{x} \rho_{R}+\lambda_{y} \rho_{B}\right)}{\rho_{B} \rho_{R}\left(1+\gamma_{F}\right)\left(R_{F}-2 \tau_{F}^{T}\right)}, \\
& \alpha_{B}^{D F^{o p t}} \simeq \frac{\gamma_{F} \lambda_{y} \rho_{B}-\left(\alpha_{R}+\alpha_{R} \gamma_{F}-1\right)\left(\gamma_{F} \lambda_{x}+\left(\frac{2 \tilde{\tau}}{R_{F}}-1\right) \rho_{B}\right) \rho_{R}}{\rho_{B}\left(1+\gamma_{F}\right)\left(\gamma_{F} \lambda_{y}-\rho_{R}\left(\frac{2 \tilde{\tau}}{R_{F}}-1\right)\left(\alpha_{R}+\alpha_{R} \gamma_{F}-1\right)\right)} .
\end{aligned}
$$

The NU throughput for AF and DF relaying schemes can be obtained by substituting $\alpha_{B}=\alpha_{B}^{i \text { opt }}$ into expressions for $\tau_{N}^{i}$. Clearly, $\tau_{N}^{i}$ is now a function only of symbol rates $R_{N}$ and $R_{F}$ (and therefore $\gamma_{N}$ and $\gamma_{F}$ ). Thus, careful choice of symbol rates is essential for maximizing the NU throughput. Let $R_{N}^{o p t}$ and $R_{F}^{o p t}$ denote the optimal symbol rates that maximize $\tau_{N}^{i}$. Now, the optimization problem is formulated as

$$
R_{N}^{\text {opt }}, R_{F}^{\text {opt }}=\arg \max _{R_{N}, R_{F}} \tau_{N}^{i}\left(R_{N}, R_{F}\right) \quad \text { such that } \alpha=\alpha_{B}^{i o p t} .
$$

Solving the above joint optimization problem analytically is difficult. However, numerical techniques can be used to pick the optimal $R_{N}$ and $R_{F}$.

In the presence of the $\mathrm{B}-\mathrm{U}_{\mathrm{F}}$ link, a three-dimensional search is required to determine $\alpha_{B}, R_{N}$ and $R_{F}$ while ensuring that $\tau_{F}^{i} \geq \tilde{\tau}$.

$\mathrm{EE}$ is an important performance metric for any communication system. It is defined as [16]:

$$
\eta_{E}^{i}=\frac{\text { Sum throughput }}{\text { Total energy consumed }}, \quad i \in\{A F, D F\},
$$

where 'sum throughput' represents the sum of $\tau_{N}^{i}$ and $\tau_{F}^{i}$. Let $T$ denote the signalling duration. Let $E_{B}=\frac{P_{B} T^{N}}{2}$ and $E_{R}=$ $\frac{P_{R} T}{2}$. Using $\tau_{N}^{i}$, and $\tau_{F}^{i}$ from (50), the EE of the system is expressed as

$$
\eta_{E}^{i}=\frac{\tau_{\text {sum }}^{i}}{E_{B}+E_{R}}, \quad \text { where } \quad \tau_{\text {sum }}^{i}=\tau_{F}^{i}+\tau_{N}^{i} .
$$

Since the sum throughput $\tau_{\text {sum }}^{i}$ is function of symbol rates $R_{N}$ and $R_{F}$, careful choice of symbol rates is essential for maximizing the EE. Let $R_{N}^{\text {opt }}$ and $R_{F}^{\text {opt }}$ denote the optimal symbol rates that maximize $\eta_{E}^{i}$. The EE optimization problem can be formulated as

$$
R_{N}^{o p t}, R_{F}^{o p t}=\arg \max _{R_{N}, R_{F}} \eta_{E}^{i}\left(R_{N}, R_{F}\right)
$$

Obtaining a closed-form solution to the above joint optimization problem is extremely difficult. However, a twodimensional search can be used to find the optimal $R_{N}$ and $R_{F}$.

\section{Simulations and Numerical Results}

In this section we present computer simulations to confirm accuracy of the derived analytical expressions and derive useful insights. Unless mentioned otherwise, the considered system parameters are as follows: $d_{B R}=d_{R F}=d_{R N}=2$,
$d_{B N}=1.2, d_{B F}=4$, and $m=4$. Also $\sigma^{2}$ is normalized to unity [17]. Further, we assume that $P_{B}=P_{R}=P\left(\rho_{B}=\rho_{R}=\right.$

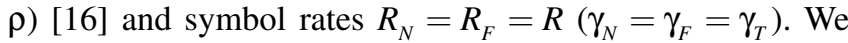
also compare the performance of the proposed framework with its relayed OMA counterpart described in Section II

In Fig 2 and Fig 3 , we plot $p_{N}^{A F}$ and $p_{F}^{A F}$ versus transmit SNR $(\rho)$ for different values of $\alpha_{B}$ and $R$. The accuracy of derived analytical expressions in (26), (28) and (35) can be clearly confirmed by simulations. Also the derived highSNR approximations in 30 and 27) are quite accurate. With decreasing $\alpha_{B}$, more power is allocated to $\mathrm{U}_{\mathrm{F}}$, and $p_{F}^{A F}$ therefore continuously improves as shown in Fig 2 It is evident from Fig 3 that an initial increase in $\alpha_{B}$ significantly improves $p_{N}^{A F}$ due to successful decoding of $x_{F}$ and $x_{N}$ at $\mathrm{U}_{\mathrm{N}}$, while further increase in $\alpha_{B}$ deteriorates $p_{N}^{A F}$ as $\mathrm{U}_{\mathrm{N}}$ fails to decode $x_{F}$. From Fig 2 and Fig 3 it is clear that (unlike in all CDRT schemes proposed so far) combining of incoming signals from $B$ and $R$ at $U_{N}$ as well as $U_{F}$ in the proposed CDRT framework ensures a full diversity of 2 to be harnessed (as derived in Lemma 3 for the case of AF relaying) by both users. However, in the absence of the $B-U_{F}$ link, a diversity order of 1 is attained at $\mathrm{FU}$ (as noted in Remark 3). The switching of diversity order from 2 to 1 and 0 can also be observed when changing $\alpha_{B}$ from 0.1, 0.3 , and 0.4 for $R=2$. Therefore, a careful choice of power allocation coefficient is important to attain full diversity for both users.

Fig 4 and Fig 5 depict the variation of $p_{N}^{D F}$ and $p_{F}^{D F}$ versus $\rho$ for different values of $\alpha_{B}, \alpha_{R}$ and $R$. Simulations confirm the accuracy of the analytical expressions derived in (40), (43) and 46 respectively. Also, the approximate high-SNR expressions for outage probability derived in (44) and (41) are quite accurate. A decrease in $\alpha_{B}$ and $\alpha_{R}$ signifies that more power is allocated to $\mathrm{U}_{\mathrm{F}}$, and $p_{F}^{D F}$ therefore continuously improves with decreasing $\alpha_{B}$ and $\alpha_{R}$ as shown in Fig 4. Clearly, from Fig $5, p_{N}^{D F}$ significantly improves with increasing $\alpha_{B}$ initially (due to successful decoding of $x_{F}$ and $x_{N}$ at $\mathrm{U}_{\mathrm{N}}$ ), while further increase in $\alpha_{B}$ degrades $p_{N}^{A F}\left(\mathrm{U}_{\mathrm{N}}\right.$ fails to decode $\left.x_{F}\right)$. Similar to the case of AF relaying, full diversity order of two can be attained (derived in Lemma 6 for both $\mathrm{U}_{\mathrm{N}}$ and $\mathrm{U}_{\mathrm{F}}$ in the presence of the direct link. While in the absence of a direct link to $U_{F}$, a diversity of order 1 can be achieved (as noted in Remark 5), while a diversity of 2 is achieved by $\mathrm{U}_{\mathrm{N}}$.

Fig 6 shows the variation of $\tau_{F}^{i}$ versus $\rho$ at $R=1,2$. Here, we compare both DF and AF relaying schemes considering optimal power allocation coefficients $\alpha_{B}$ and $\alpha_{R}$. In the presence of the $B-\mathrm{U}_{\mathrm{F}}$ link, performance with $\mathrm{DF}$ is superior to that of AF for lower SNRs. At high-SNRs, the performance of both $\mathrm{AF}$ and $\mathrm{DF}$ is comparable. However, in the absence of the $B-\mathrm{U}_{\mathrm{F}}$ link, performance with $\mathrm{DF}$ is always superior. AF relays are often preferred due to their simplicity and can be seen to result in good performance except at low SNRs. Fig 7 depicts $\tau_{N}^{i}$ versus $\rho$ for $R=1,2$. For a fair comparison of $\mathrm{AF}$ and DF relaying, optimal power allocation coefficients are used at B and R. We observe 


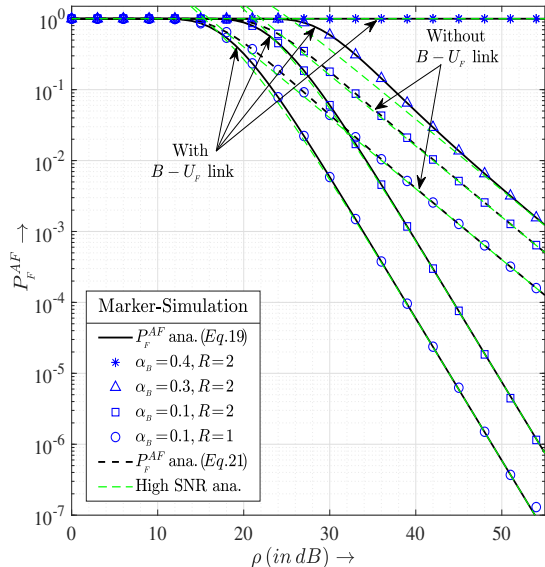

Fig. 2: $p_{F}^{A F}$ vs. $\rho$.

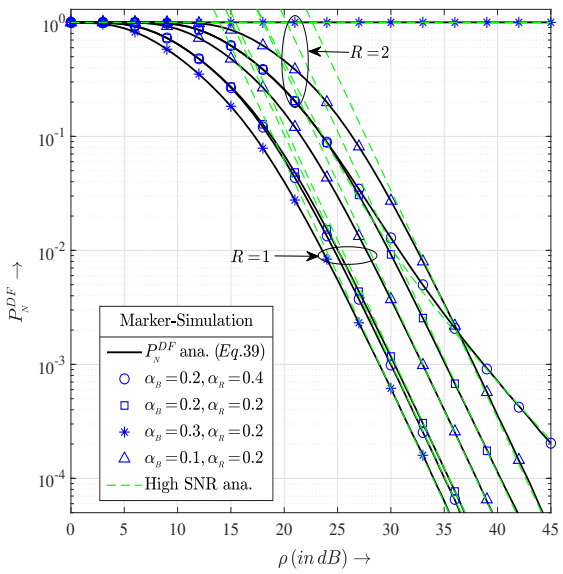

Fig. 5: $p_{N}^{D F}$ vs. $\rho$.

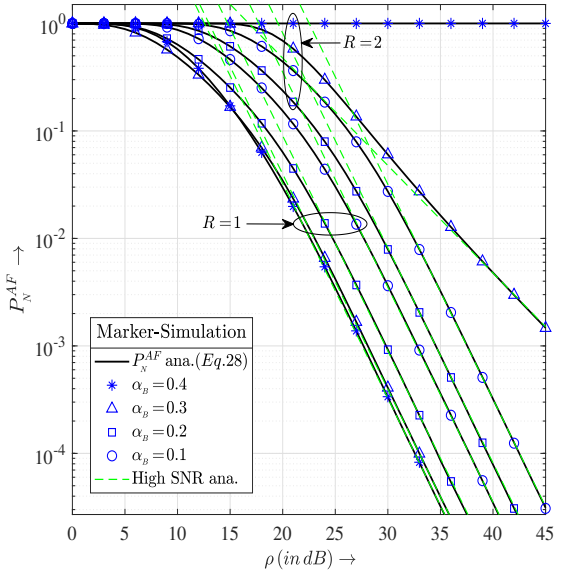

Fig. 3: $p_{N}^{A F}$ vs. $\rho$.

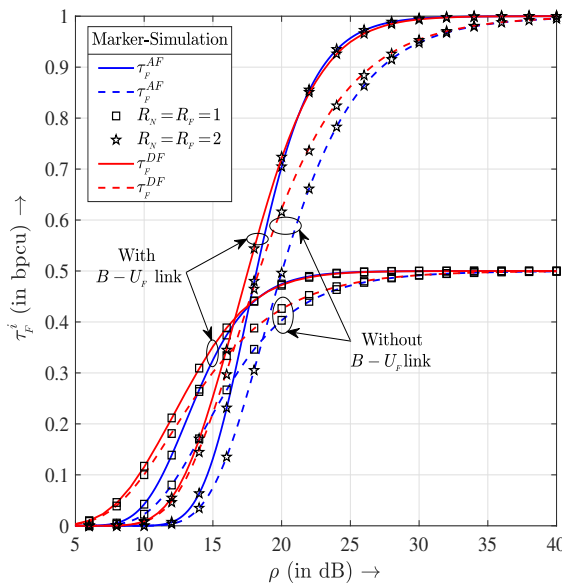

Fig. 6: $\tau_{F}^{A F}$ or $\tau_{F}^{D F}$ vs. $\rho$.

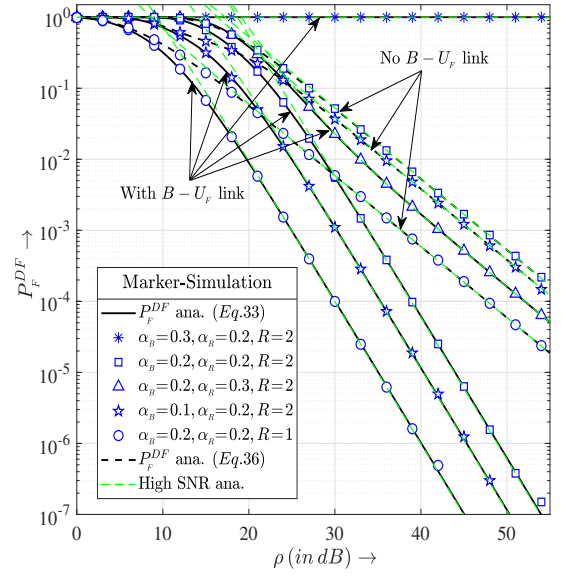

Fig. 4: $p_{F}^{D F}$ vs. $\rho$.

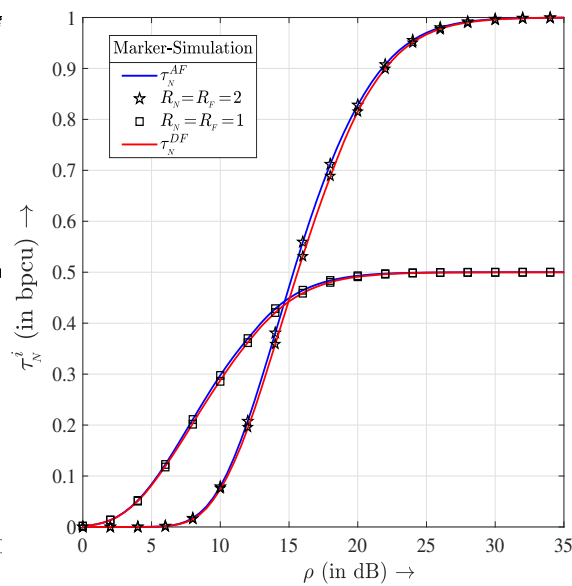

Fig. 7: $\tau_{N}^{A F}$ or $\tau_{N}^{D F}$ vs. $\rho$. that both $\mathrm{AF}$ and $\mathrm{DF}$ achieve almost similar performance in terms of NU throughpur ${ }^{4}$

Fig 8 depicts the variation of $\tau_{N}^{A F}$ or $\tau_{F}^{A F}$ versus $\alpha_{B}$ for $R=2,3$. When $\alpha_{B}$ is very small, more power is allocated to $\mathrm{U}_{\mathrm{F}}$ and $\tau_{F}^{A F}$ therefore is maximum at $\alpha_{B}=0$. With increase in $\alpha_{B}, \tau_{F}^{A F}$ gradually decreases and becomes 0 for $\alpha_{B} \geq \frac{2}{2+\gamma_{F}}$ due to unsuccessful decoding of $s_{F}$ at $\mathrm{U}_{\mathrm{F}}$. However, in the absence of the $B-\mathrm{U}_{\mathrm{F}}$ link, $\tau_{F}^{A F}$ becomes zero for $\alpha_{B} \geq \frac{1}{1+\gamma_{F}}$. Therefore, the presence of $B-\mathrm{U}_{\mathrm{F}}$ link significantly improves the FU performance and allows a wider range of valid power allocations. On the other hand $\tau_{N}^{A F}$ increases initially and attains a maximum value (at $\alpha_{B}=\frac{1}{1+\gamma_{F}}$ ). Further increase in $\alpha_{B}$ decreases the probability of successful decoding of $s_{F}$ at $\mathrm{U}_{\mathrm{N}}$, and $\tau_{N}^{A F}$ therefore also decreases.

In Fig 9. $\tau_{N}^{D F}$ or $\tau_{F}^{D F}$ is plotted versus $\alpha_{B}$ (considering optimal $\alpha_{R}$ ) and $\alpha_{R}$ (considering optimal $\alpha_{B}$ ) for $R=1.5,2.5$. As with AF relaying, $\tau_{F}^{D F}$ is maximum at $\alpha_{B}=0$ and then decreases to 0 with increasing $\alpha_{B}$. $\tau_{N}^{D F}$ increases initially, attains a maximum value at $\alpha_{B}=\frac{\gamma_{N}}{\gamma_{N}+\gamma_{F}+\gamma_{F} \gamma_{N}}$, and then

\footnotetext{
${ }^{4}$ Although DF relay $\mathrm{R}$ offers an additional degree of freedom in terms of $\alpha_{R}$ (power allocation at $\mathrm{R}$ ), $\mathrm{AF}$ achieves performance almost similar to it. This is because in CDRT-NOMA with DF relaying, power allocation at $\mathrm{R}$ takes place if and only if R decodes both FU and NU symbols successfully.
}

decreases to 0 (the SINR to decode $s_{F}$ at NU decreases). It can be observed that the presence of the $B-\mathrm{U}_{\mathrm{F}}$ significantly improves the FU throughput. Also, both $\tau_{F}^{D F}$ and $\tau_{N}^{D F}$ change significantly with $\alpha_{B}$ while the change in $\alpha_{R}$ does not significantly change the throughpu 5 Hence, an optimal choice of $\alpha_{B}$ is important. For this reason, we study the variation of EE w.r.t $\alpha_{B}$ only. From Fig 8 and Fig 9 , it can be clearly seen that CDRT-NOMA with $\mathrm{AF}$ relaying provides a wider range of valid power allocations in comparison to DF relaying for both $\mathrm{U}_{\mathrm{N}}$ and $\mathrm{U}_{\mathrm{F}}$. The presence of the $B-\mathrm{U}_{\mathrm{F}}$ widens the valid range of power allocations for $U_{F}$ with $A F$ relaying (but not with DF relaying).

Fig 10 shows the variation of NU and FU throughout with respect to the $d_{B N}$ to $d_{B F}$ ratio (we vary $d_{B N}$ while fixing $d_{B F}=4$ to vary $\frac{d_{B N}}{d_{B F}}$ ) for AF and DF relaying, respectively. For AF as well as DF relaying, FU throughput is constant due to fixed $d_{B F}$, whereas $\mathrm{NU}$ throughput increases with increasing $d_{B N}$ to $d_{B F}$ ratio and saturates after a certain value due to the fixed target rate. Also, for lower distance ratios, the considered CDRT-NOMA with DF relaying performs

\footnotetext{
${ }^{5}$ Since transmission from R depends on successful decoding of $s_{F}$, power allocation at $\mathrm{S}$ is crucial. Also, at $\mathrm{R}$, power allocation is required only if both $s_{F}$ and $s_{N}$ get successfully decoded. Therefore, an optimal choice of $\alpha_{B}$ is more important in comparison to $\alpha_{R}$.
} 


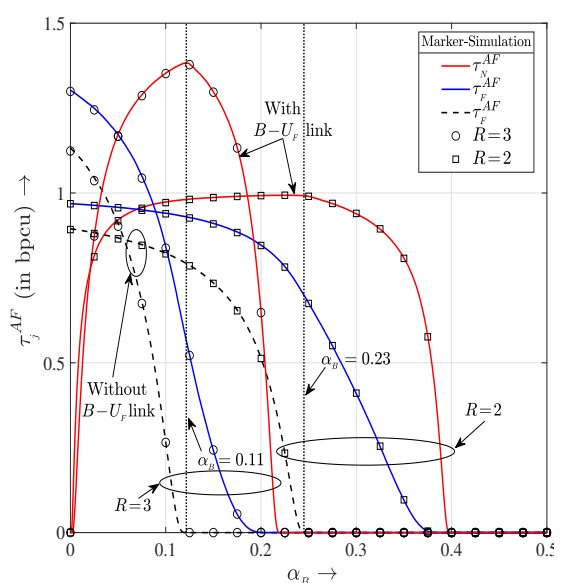

Fig. 8: $\tau_{N}^{A F}$ or $\tau_{F}^{A F}$ vs. $\alpha_{B}$ with $\rho=30 \mathrm{~dB}$.

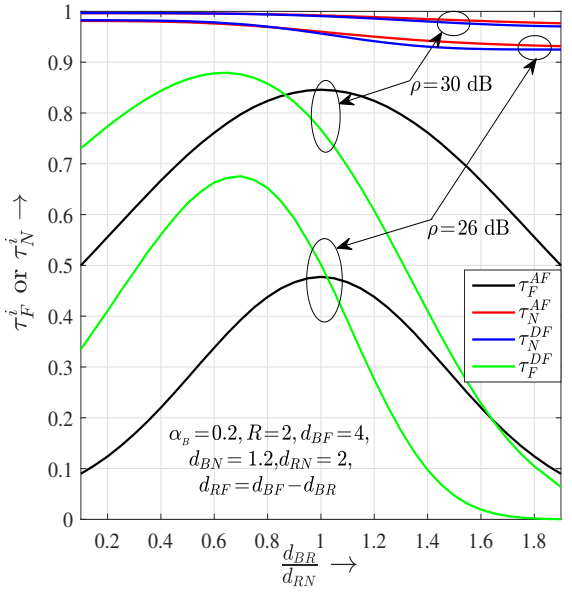

Fig. 11: $\tau_{N}^{A F}$ or $\tau_{F}^{A F}$ vs. the ratio of $d_{B R}$ to $d_{R N}$.

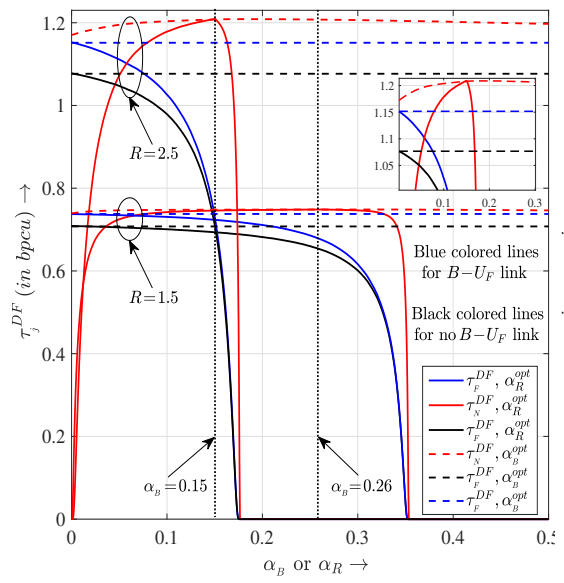

Fig. 9: $\tau_{N}^{D F}$ or $\tau_{F}^{D F}$ vs. $\alpha_{B}$ with $\rho=30 \mathrm{~dB}$.

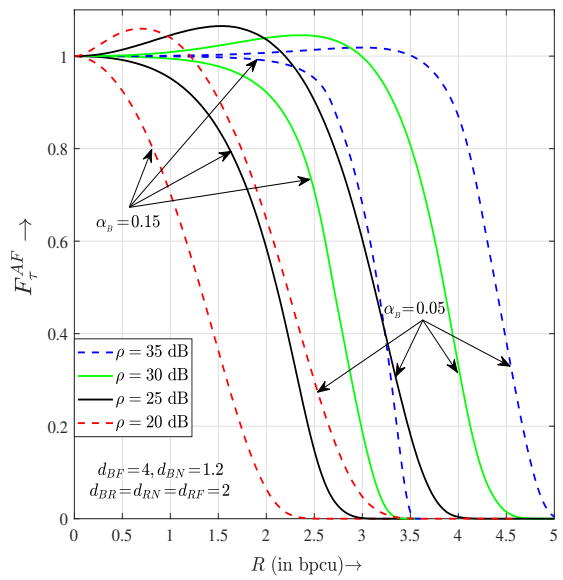

Fig. 12: $F_{\tau}^{A F}$ vs. $R$ for different $\alpha_{B}$ and $\rho$.

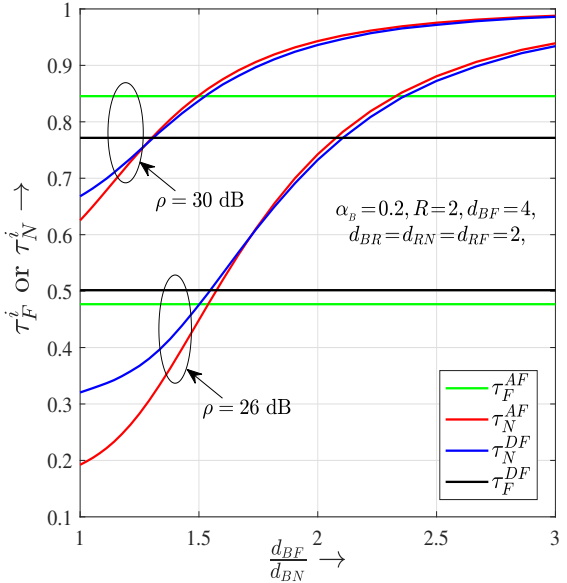

Fig. 10: $\tau_{N}^{A F}$ or $\tau_{F}^{A F}$ vs. the ratio of $d_{B N}$ to $d_{B F}$.

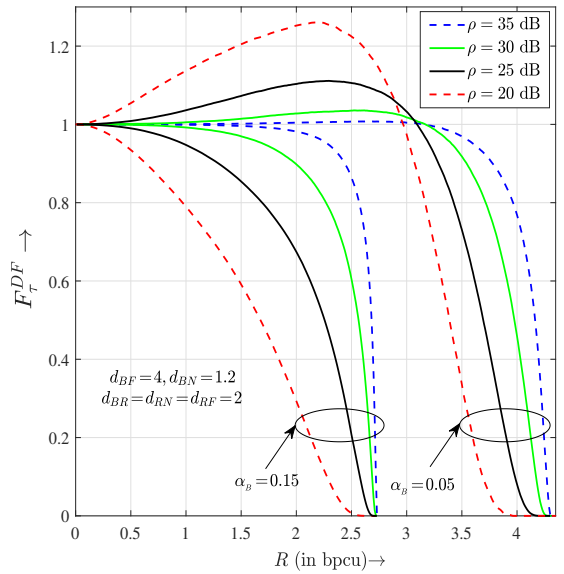

Fig. 13: $F_{\tau}^{D F}$ vs. $R$ for different $\alpha_{B}$ and $\rho$ better than with AF relaying, whereas both $\mathrm{DF}$ and $\mathrm{AF}$ show almost similar performance for high distance ratios.

Fig 11 depicts the variation of NU and FU throughput with respect to the $d_{B R}$ to $d_{R N}$ ratio (to vary $\frac{d_{B R}}{d_{R N}}$, we vary $d_{B R}$ while fixing $d_{R N}=2$ ) for AF and DF relaying, respectively. In both the relaying schemes, the FU throughput initially increases with increasing $\frac{d_{B R}}{d_{R N}}$, attains a maximum value, and then decreases to zero. Therefore, the relay location significantly impacts the FU's performance.

Fig 12 and Fig 13 depict user fairness $\sqrt[6]{6}$ versus target rate of AF and DF relay-based CDRT-NOMA, respectively. User fairness depends on the choice of target rate and NOMA power allocation factor. In both cases, the fairness decreases with increasing $\alpha_{B}$. On the other hand, increasing transmit SNR results in increased fairness. Also, high transmit SNR ensures a wider range of valid target rates to maintain user

\footnotetext{
${ }^{6}$ In NOMA signalling, due to apportioning of power between users, the user performance differs and, a user may have better performance in comparison to others. To maintain user fairness, the performance gap among users should not be large. Similar to [13], we also define the proportional fairness in terms of the ratio of $\mathrm{FU}$ to $\mathrm{NU}$ throughput as $F_{\tau}^{i}=\frac{\tau_{F}^{i}}{\tau_{N}^{i}}$ where $i \in\{A F, D F\}$.
}

fairness.

In Fig 14, we plot the sum throughput $\tau_{\text {sum }}^{i}=\tau_{F}^{i}+\tau_{N}^{i}$ versus $R=R_{N}=R_{F}$ using optimal $\alpha_{B}$ and $\alpha_{R}$. With increase in SNR $\tau_{\text {sum }}^{i}$ also increases and $\tau_{\text {sum }}^{i}$ is a quasi-concave function of $R$, and a unique value $R^{\text {opt }}$ exists at which $\tau_{\text {sum }}^{i}$ is maximum. Clearly, CDRT-NOMA with DF outperforms that with $\mathrm{AF}$ in terms of sum throughput.

Fig 15 illustrates the effect of transmit power $\left(P_{B}=P_{R}=\right.$ $P)$ on the EE of AF or DF relay-aided CDRT-NOMA. It can be observed that at $\rho=30 \mathrm{~dB}$ and $R=2$, with $P=2 W$ $\eta_{E}^{A F}=0.955$ and $\eta_{E}^{D F}=0.945$, while at $P=3 W, \eta_{E}^{A F}=$ 0.637 and $\eta_{E}^{D F}=0.630$. Thus, EE improves with decreasing $P$. In addition to this, we observe that for the mid-SNR range, DF outperforms AF, while at low as well as at highSNRs, both achieve similar performance in terms of EE. However, in the absence of the $B-\mathrm{U}_{\mathrm{F}}$ link, DF outperforms AF for a wider range of SNRs, and the gap is more than when the $B-\mathrm{U}_{\mathrm{F}}$ link is present.

In Fig 16, we plot $\eta_{E}^{A F}$ and $\eta_{E}^{D F}$ versus $\alpha_{B}$ with $R=1$ and $\rho=30 \mathrm{~dB}$ for $P=1 W$ and $P=2 W$. We also compare the EE of CDRT-NOMA with its relayed OMA counterpart. It is evident that EE of both AF and DF relay-aided NOMA 


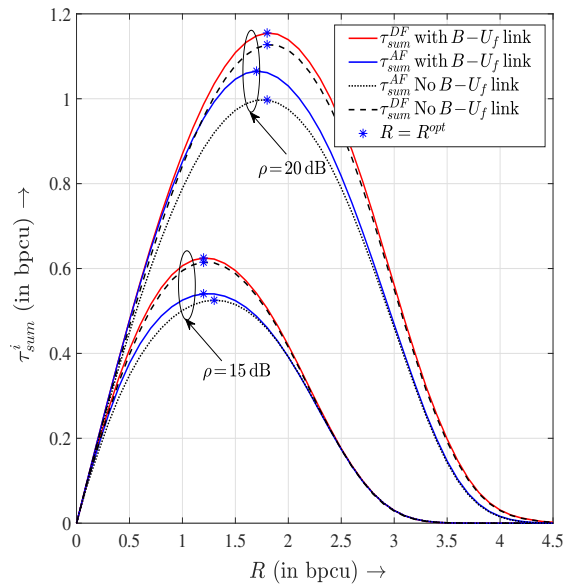

Fig. 14: $\tau_{\text {sum }}^{A F}$ or $\tau_{\text {sum }}^{D F}$ vs. $R$.

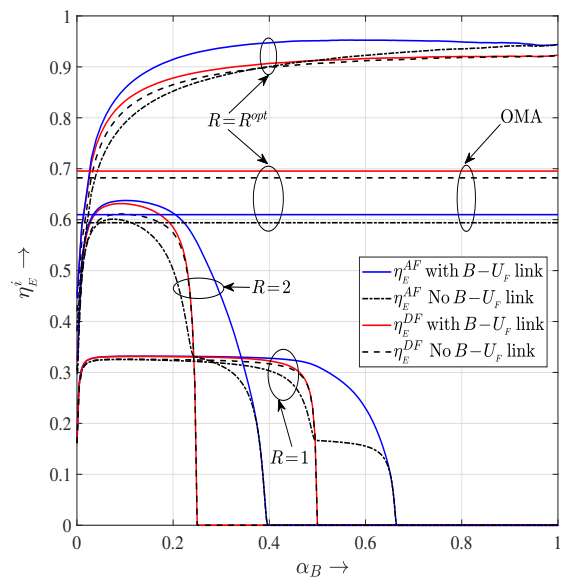

Fig. 17: $\eta_{E}^{A F}$ or $\eta_{E}^{D F}$ vs. $\alpha_{B}$.

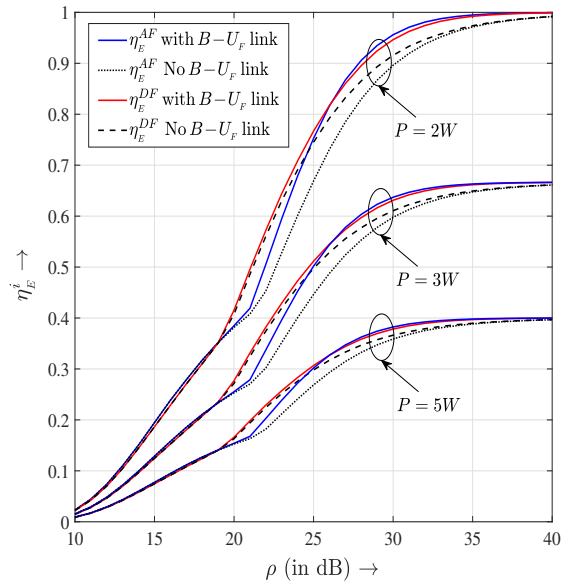

Fig. $15: \eta_{E}^{A F}$ or $\eta_{E}^{D F}$ vs. $\rho$.

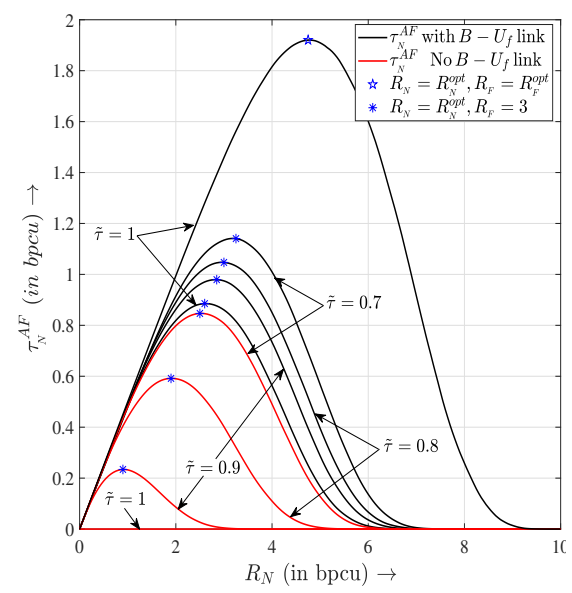

Fig. 18: $\tau_{N}^{A F}$ vs. $R_{N}$.

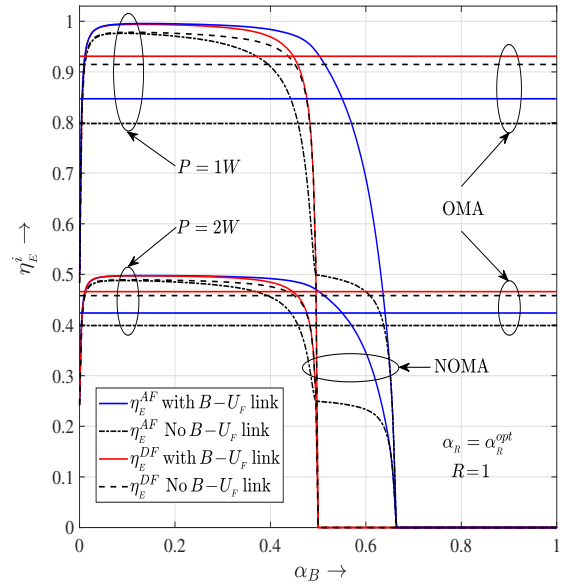

Fig. 16: $\eta_{E}^{A F}$ or $\eta_{E}^{D F}$ vs. $\alpha_{B}$.

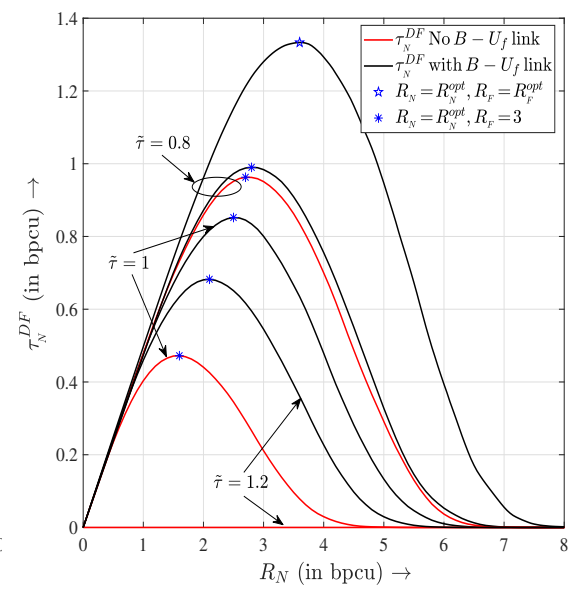

Fig. 19: $\tau_{N}^{D F}$ vs. $R_{N}$. in CDRT is higher than that of relayed OMA. This is large because the signals from both $\mathrm{B}$ and $\mathrm{R}$ are combined at NU and FU. It can be seen that in the OMA case, EE with DF is better than that with AF, whereas in CDRT-NOMA, AF is more energy efficient. We also show that the $B-\mathrm{U}_{\mathrm{F}}$ link significantly improves the EE (the gap is larger with $\mathrm{AF}$ than DF).

In Fig. 17. we plot the variation of $\eta_{E}^{A F}$ and $\eta_{E}^{D F}$ versus $\alpha_{B}$ for fixed transmit power $(P=3 W), \rho=30 \mathrm{~dB}$ and $R=$ $1,2, R^{\text {opt }}$. Clearly, the energy efficiencies of AF as well as DF increase as we increase the symbol rate from 1 to 2 , and the improvement is huge when the optimal symbol rate $\left(R^{o p t}\right)$ is used. Further, this is true for the entire range of the power allocation parameter. We also observe that EE of relay-aided CDRT-NOMA is significantly higher than that of OMA.

Fig 18 and Fig 19 depict $\tau_{N}^{A F}$ and $\tau_{N}^{D F}$ versus $R_{N}$ for NOMA in CDRT with AF and DF relaying, respectively for different $\tau_{F}=\tilde{\tau}$ and $\rho=25 \mathrm{~dB}$. Fig 18 and Fig 19 are plotted for optimum values of $\alpha_{B}$ derived in (51) and (52), respectively. It can be seen that $\tau_{N}$ increases with decreasing $\tilde{\tau}$. In case of both DF and AF aided NOMA in CDRT, we observe that by optimally choosing $R_{N}$, the NU throughput can be maximized while guaranteeing the desired FU throughput $\tilde{\tau}$. Also, selecting an optimal rate $\left(R_{F}=R_{F}^{\text {opt }}\right.$ ) helps to provide huge gain in NU throughput in comparison to any fixed rate $R_{F}$. Therefore, the NU throughput attains a maximum performance at $\left(R_{N}^{o p t}, R_{F}^{o p t}\right)$. Clearly, jointly optimizing $R_{N}$ and $R_{F}$ (as in Section IV] is of utmost importance. It can also be observed that presence of the $B-\mathrm{U}_{\mathrm{F}}$ significantly improves the NU performance.

In Fig 20, Fig 21 and Fig 22, we compareSince the SDF CDRT scheme (with 2-bit feedback) presented in [26] linearly combines the first and second phase signals with orthogonal power allocation at BS and R. FU symbols are canceled at NU, and both the users recover their desired symbols. Due to this, no SIC is required at NU, and analytical complexity is reduced. However, the scheme is sub-optimal and results in a diversity loss for both users. On the other hand, our framework requires no feedback and uses combining at both users with SIC at NU. This results in a diversity order of 2 with improved performance at both the users, but with a minor increase in complexity for combining. the performance of proposed AF as well as DF relay-aided CDRT-NOMA with SDF CDRT-NOMA of [26] in terms of outage probability, sum throughput, and $\mathrm{EE}$, respectively. It is clear from Fig 20 that for optimal power allocation, the proposed AF, as well as DF relay-aided 


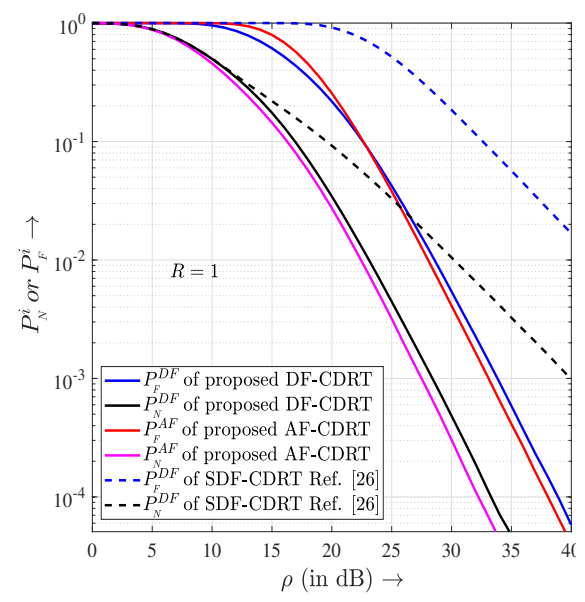

Fig. 20: $P_{N}^{i}$ or $P_{F}^{i}$ vs. $\rho$.

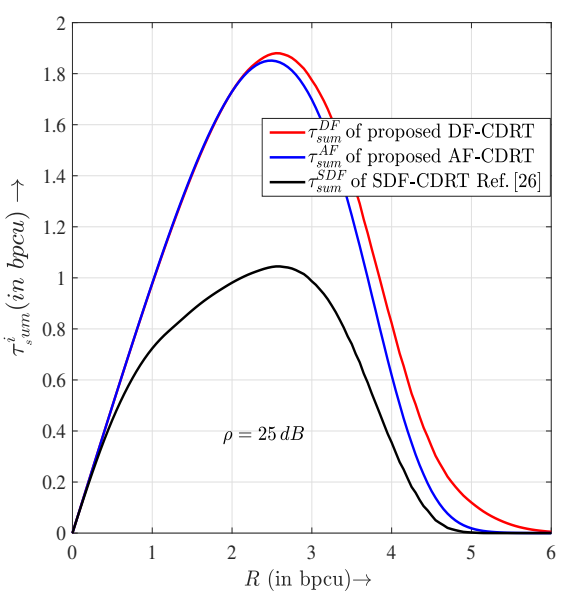

Fig. 21: $\tau_{\text {sum }}^{i}$ vs. $R$.

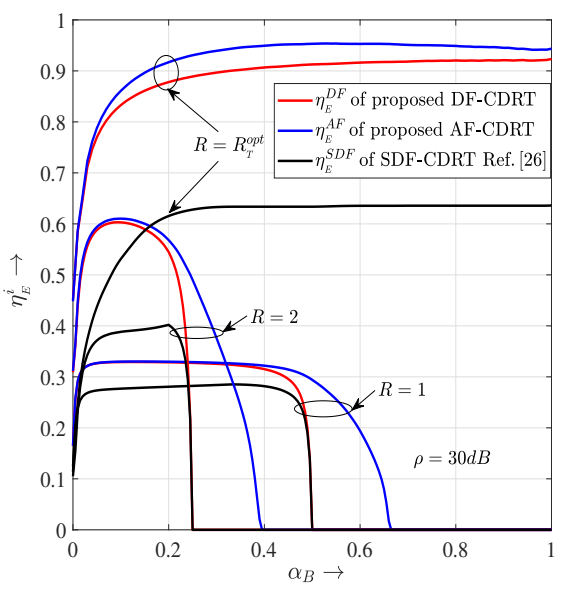

Fig. 22: $\eta_{E}^{i}$ vs. $\alpha_{B}$.
CDRT-NOMA, outperform the SDF CDRT-NOMA scheme of [26] by a huge margin. The SDF CDRT-NOMA achieves a diversity order of one, whereas the proposed framework can achieve a diversity of two at both the users. It is clear from Fig 21 that the proposed almost doubles the sum throughput in comparison to the SDF CDRT-NOMA scheme of [26]. Fig 22 illustrates that the proposed framework is also more energy-efficient.

\section{CONCLUSION}

In this paper, we analyzed the performance of a new framework for downlink non-orthogonal multiple access (NOMA) in a coordinated direct and relay transmission (CDRT) scheme with direct links to the near-user (NU) and the far-user (FU). In this framework, NU combines the direct and relayed signals while performing successive interference cancellation, which considerably improves its outage performance. Considering either amplify-andforward (AF) or decode-and-forward (DF) relaying, closedform expressions for outage probability and throughput were derived for both NU and FU. Further, we have also derived the high-SNR approximations to the outage probability and shown that the proposed framework enables both NU and FU to attain a diversity of two without feedback, which is a significant advancement over all existing schemes. This scheme is very useful in multimedia and other applications where the users have stringent outage constraints. It was also demonstrated that the performance of NU with $\mathrm{AF}$ and DF relaying is similar in the entire SNR range. For $\mathrm{FU}$, in the presence of the BS-FU link, DF outperformed $\mathrm{AF}$ at low SNRs while at high SNRs, both resulted in similar performance. However, DF always outperformed AF in the absence of the BS-FU link. Further, we demonstrated how the choice of power allocation coefficient and target symbol rates are crucial to attain maximum NU throughput while guaranteeing the desired target throughput at FU. We compared the performance of the proposed framework with selective decode-and-forward (SDF) CDRT-NOMA in terms of three metrics: outage probability, sum throughput, and energy efficiency (EE) and demonstrated that the proposed framework, without using any feedback, provides huge performance gain in terms of all three metrics and helps to secure an additional diversity order at both the users. Furthermore, by comparing the performance of the proposed CDRT based NOMA system to its OMA counterpart, we demonstrated that the proposed system provides higher EE, and the presence of BS-FU link significantly improves the system performance. We also demonstrated that optimal rate selection is essential to maximize $\mathrm{EE}$.

\section{APPENDIX A}

Proof of Theorem 7 . Substituting $\zeta_{F_{1}}(w)$ from line following (20) into 24], $I_{1}^{-}$can be expressed as

$$
\begin{aligned}
I_{1} & =\int_{0}^{\chi_{F}} \lambda_{w} \exp \left(\frac{-\xi_{1}\left(w-\chi_{F}\right)\left(\lambda_{x} \rho_{R}+\lambda_{y} \rho_{B}\right)}{\rho_{R}\left(w-\xi_{1}\right)}-\lambda_{w} w\right) \\
& \times 2 \sqrt{\frac{-\xi_{1}\left(w-\chi_{F}\right) \lambda_{x} \lambda_{y}}{\rho_{R}\left(w-\xi_{1}\right)}} K_{1}\left(2 \sqrt{\frac{-\xi_{1}\left(w-\chi_{F}\right) \lambda_{x} \lambda_{y}}{\rho_{R}\left(w-\xi_{1}\right)}}\right) d w .
\end{aligned}
$$

For large values of $\rho_{B}$ and $\rho_{R}$, the argument inside the Bessel function becomes very small throughout the range of SNRs (as verified in Section $\mathrm{V}$ ). We therefore use $K_{1}(\theta) \simeq \frac{1}{2} \Gamma(1)\left(\frac{\theta}{2}\right)^{-1}[35,9.6 .9]$. Using $\lambda_{w}\left(w-\xi_{1}\right)=w$ we obtain

$I_{1} \simeq e^{-\xi_{1}\left(\lambda_{w}+\frac{1}{\rho_{R}}\right)} \int_{-\xi_{1} \lambda_{w}}^{\lambda_{w}\left(\chi_{F}-\xi_{1}\right)} \exp \left(-w-\frac{\lambda_{w} \xi_{1}\left(\xi_{1}-\chi_{F}\right)\left(\lambda_{x} \rho_{R}+\lambda_{y} \rho_{B}\right)}{\rho_{R} w}\right) d w$.

Using $\int^{\infty} t^{a-1} e^{-t-\frac{b}{t}} d t=\Gamma(a, x ; b)[36$. eq. (4)] in the above, we obtain

$I_{1} \simeq e^{-\xi_{1}\left(\lambda_{w}+\frac{1}{\rho_{R}}\right)}\left[\Gamma\left(1, \xi_{1} \lambda_{w} ; \vartheta_{6}\right)-\Gamma\left(1, \lambda_{w}\left(\chi_{F}-\xi_{1}\right) ; \vartheta_{6}\right)\right],(5 \vartheta)$

where $\vartheta_{6}=\frac{\lambda_{w} \xi_{1}\left(\xi_{1}-\chi_{F}\right)\left(\lambda_{x} \rho_{R}+\lambda_{y} \rho_{B}\right)}{\rho_{R}}$. Similarly, we solve for $I_{2}$ as

$I_{2} \simeq e^{-\xi_{1}\left(\lambda_{w}+\frac{1}{\rho_{R}}\right)} \int_{0}^{\infty} \exp \left(-w-\frac{\lambda_{w} \xi_{1}\left(\xi_{1}-\chi_{F}\right)\left(\lambda_{x} \rho_{R}+\lambda_{y} \rho_{B}\right)}{\rho_{R} w}\right) d w$. 
Using $\int_{0}^{\infty} \exp \left(-\frac{b}{4 t}-a t\right) d t=\sqrt{\frac{b}{a}} K_{1}(\sqrt{a b}) \quad$ |34, 3.324.1] in the above, we obtain

$$
I_{2} \simeq e^{-\xi_{1}\left(\lambda_{w}+\frac{1}{\rho_{R}}\right)} \sqrt{4 \vartheta_{6}} K_{1}\left(\sqrt{4 \vartheta_{6}}\right) .
$$

After substituting $I_{1}$ and $I_{2}$ from (59) and (61) into (21), we obtain (26).

\section{APPENDIX B}

Proof of Lemma 17. After some mathematical arrangements, 23 can be expressed as

$$
\begin{aligned}
I_{1} & =\int_{o}^{\chi_{F}} \exp \left(-\zeta_{F_{1}}(w)\left(\lambda_{x}+\frac{\lambda_{y} \rho_{B}}{\rho_{R}}\right)\right) \\
& \times \int_{0}^{\infty} \lambda_{x} \frac{\exp \left(-\lambda_{x} x\right)}{\exp \left(\frac{\lambda_{y} \zeta_{F_{1}}(w)\left[1+\rho_{B} \zeta_{F_{1}}(w)\right]}{x \rho_{R}}\right)} \lambda_{w} e^{-\lambda_{w} w} d x d w .
\end{aligned}
$$

For large $\rho_{B}$ and $\rho_{R}$, we use the linear approximation to the exponential term in the denominator to obtain

$$
\begin{aligned}
I_{1} & \simeq \int_{o}^{\chi_{F}} \exp \left(-\zeta_{F_{1}}(w)\left(\lambda_{x}+\frac{\lambda_{y} \rho_{B}}{\rho_{R}}\right)\right)\left[\int_{0}^{\infty} \lambda_{x} e^{-\lambda_{x} x} d x\right. \\
& \left.-\xi_{2}(w) \int_{0}^{\infty} \frac{\lambda_{x} e^{-\lambda_{x} x}}{x+\xi_{2}(w)} d x\right] \lambda_{w} e^{-\lambda_{w} w} d w,
\end{aligned}
$$

where $\xi_{2}(w) \simeq \frac{\lambda_{y} \zeta_{F_{1}}(w)\left[1+\rho_{B} \zeta_{F_{1}}(w)\right]}{\rho_{B}}$. Using [34, 3.352.6] in the above, we obtain

$$
\begin{gathered}
I_{1} \simeq \int_{o}^{\chi_{F}} \exp \left(-\zeta_{F_{1}}(w)\left(\lambda_{x}+\frac{\lambda_{y} \rho_{B}}{\rho_{R}}\right)\right)\left(1-\xi_{2}(w) \lambda_{x}\right. \\
\left.\times e^{\xi_{2}(w) \lambda_{x}} \mathcal{E}_{1}\left(\xi_{2}(w) \lambda_{x}\right)\right) \lambda_{w} e^{-\lambda_{w} w} d w .
\end{gathered}
$$

Further using $e^{\xi_{2}(w) \lambda_{x}} \mathcal{E}_{1}\left(\xi_{2}(w) \lambda_{x}\right)=\frac{1}{1+\xi_{2}(w) \lambda_{x}}[35,5.1 .19]$ and simplifying, we obtain

$$
I_{1} \simeq \int_{o}^{\chi_{F}} \frac{\lambda_{w} e^{-\lambda_{w} w}}{1+\xi_{2}(w) \lambda_{x}} \exp \left(-\zeta_{F_{1}}(w)\left(\lambda_{x}+\frac{\lambda_{y} \rho_{B}}{\rho_{R}}\right)\right) d w .
$$

Substituting for $\zeta_{F_{1}}(w)$ from the line following 25 and neglecting higher-order terms of $1 / \rho_{B}$, we get

$$
\begin{aligned}
I_{1} \simeq 1-e^{-\lambda_{w} \chi_{F}}-\left(\lambda_{x}+\frac{\lambda_{y} \rho_{B}}{\rho_{R}}\right) \xi_{1}\left[\int_{o}^{\chi_{F}} \lambda_{w} e^{-\lambda_{w} w} d w\right. \\
\left.+\left(\xi_{1}-\chi_{F}\right) \lambda_{w} \int_{o}^{\chi_{F}} \frac{e^{-\lambda_{w} w}}{w-\xi_{1}} d w\right] .
\end{aligned}
$$

We use [34, 3.352.1] to obtain

$$
\begin{aligned}
& I_{1} \simeq 1-e^{-\lambda_{w} \chi_{F}}-\left(\lambda_{x}+\frac{\lambda_{y} \rho_{B}}{\rho_{R}}\right) \xi_{1}\left[1-e^{-\lambda_{w} \chi_{F}}+\left(\xi_{1}-\chi_{F}\right)\right. \\
&\left.\times \lambda_{w} e^{-\lambda_{w} \xi_{1}}\left[\mathcal{E}_{1}\left(-\lambda_{w} \xi_{1}\right)-\mathcal{E}_{1}\left(-\lambda_{w} \xi_{1}+\lambda_{w} \chi_{F}\right)\right]\right] .
\end{aligned}
$$

Similarly, the expression for $I_{2}$ is given by

$$
I_{2} \simeq \frac{e^{-\lambda_{w} \xi_{1}}}{1+\xi_{1}\left(\lambda_{x}+\lambda_{y}\right)} .
$$

After substituting for $I_{1}$ and $I_{2}$ from (67) and (68) into (21), the high-SNR approximation to $p_{F}^{A F}$ is given by (27).

\section{APPENDIX C}

Proof of Theorem 3. Using (32), $p_{N}^{A F}=1-\left(I_{5}+I_{3}\right) U(\phi)-$ $I_{4} U\left(\phi-\alpha_{B}\right)-I_{6}\left[U(\phi)-U\left(\phi-\alpha_{B}\right)\right]-I_{7} U(-\phi)$. We first solve for $I_{4}=\operatorname{Pr}\left\{V>\zeta_{N_{1}}(X, Z), X>\chi_{N_{1}}(Z), \chi_{F}<Z<\chi_{N}\right\}$ as follows:

$$
\begin{aligned}
I_{4} & =\int_{\chi_{F}}^{\chi_{N}} \int_{\chi_{N_{1}}}^{\infty} \int_{\zeta_{N_{1}}}^{\infty} f_{V}(v) f_{X}(x) f_{Z}(z) d v d x d z \\
& =\int_{\chi_{F} \chi_{N_{1}}(z)}^{\chi_{N}} \int_{x}^{\infty} \lambda_{z} \exp \left(-\lambda_{v} \zeta_{N_{1}}(x, z)-\lambda_{x} x-\lambda_{z} z\right) d x d z .
\end{aligned}
$$

Substituting $x=x-\chi_{N_{1}}(z)$ in the above and rearranging, we obtain

$$
I_{4}=\int_{\chi_{F}}^{\chi_{N}} \int_{0}^{\infty} \exp \left(-\chi_{N_{1}}(z)\left[\lambda_{x}+\frac{\lambda_{v} \rho_{B}}{\rho_{R}}\right]\right) \frac{\lambda_{x} e^{-\lambda_{x} x} \lambda_{z} e^{-\lambda_{z} z}}{\exp \left(\frac{\mu_{N_{1}}(z)}{\lambda_{x} x}\right)} d x d z
$$

where $\mu_{N_{1}}(z)=\frac{\lambda_{x} \lambda_{v} \chi_{N_{1}}(z)\left[\rho_{B} \chi_{N_{1}}(z)+1\right]}{\rho_{R}}$. Solving the above using [34, 3.324.1], we obtain

$$
I_{4}=\int_{\chi_{F}}^{\chi_{N}} e^{-\chi_{N_{1}}(z)\left(\lambda_{x}+\frac{\lambda_{\nu} \rho_{B}}{\rho_{R}}\right)} 2 \sqrt{\mu_{N 1}(z)} K_{1}\left(2 \sqrt{\mu_{N 1}(z)}\right) \lambda_{z} e^{-\lambda_{z} z} d z .
$$

The above integral does not admit a closed-form. To derive a highly accurate closed-form expression (as with $I_{1}$ ), we use $K_{1}(\theta) \simeq \frac{1}{2} \Gamma(1)\left(\frac{\theta}{2}\right)^{-1}[35,9.6 .9]$ to obtain

$$
I_{4} \simeq \lambda_{z} e^{-C_{\ell} \chi_{N}} \int_{\chi_{F}-\chi_{N}}^{0} e^{C z} d z
$$

where $C=C_{\ell}-\lambda_{z}$ with $C_{\ell}=\lambda_{x}+\frac{\lambda_{v} \rho_{B}}{\rho_{R}}$. After solving the above integral, $I_{4}$ is obtained as

$$
I_{4} \simeq \lambda_{z} e^{-\chi_{N} C_{\ell}}\left[\frac{1-e^{C\left(\chi_{F}-\chi_{N}\right)}}{C}\right] .
$$

Using a similar approach, $I_{5}, I_{6}$ and $I_{7}$ are obtained as

$$
I_{5} \simeq \frac{\lambda_{z} e^{-\chi_{N} C_{\ell}}}{C}\left[e^{C\left(\min \left(\chi_{F}, \chi_{N}\right)-\chi_{N}\right)}-e^{-C \chi_{N}}\right],
$$

$$
\begin{aligned}
& I_{6} \simeq e^{\xi_{1}\left(C_{\ell}+\lambda_{z}\right)}\left[e^{-\left(\xi_{1}+\chi_{N}\right)} e^{-\left(\xi_{1}+\chi_{F}\right)} \lambda_{z} \vartheta_{2} e^{\lambda_{z} \vartheta_{2}}\right. \\
& \left.\times\left[\mathcal{E}_{1}\left(\lambda_{z}\left(\chi_{N}+\xi_{1}+\vartheta_{2}\right)\right)-\mathcal{E}_{1}\left(\lambda_{z}\left(\chi_{F}+\xi_{1}+\vartheta_{2}\right)\right)\right]\right], \\
& I_{7} \simeq e^{\xi_{1}\left(C_{\ell}+\lambda_{z}\right)}\left[e^{-2 \xi_{1}}-\lambda_{z} \vartheta_{2} e^{\lambda_{z} \vartheta_{2}}\left[\mathcal{E}_{1}\left(\lambda_{z}\left(2 \xi_{1}+\vartheta_{2}\right)\right)\right]\right],
\end{aligned}
$$


respectively, where $\vartheta_{2}=\xi_{1}\left(\xi_{1}+\frac{\gamma_{F}}{\rho_{B} \phi}\right) C_{\ell}$. Finally, $I_{3}$ can be derived as

$$
I_{3}=\operatorname{Pr}\left\{Z>\max \left(\chi_{F}, \chi_{N}\right)\right\}=e^{-\lambda_{z} \max \left(\chi_{F}, \chi_{N}\right)} .
$$

After substituting the derived expressions of $I_{3}, I_{4}, I_{5}, I_{6}$ and $I_{7}$ from (77), (73), (74), (75) and (76), respectively, into (32), $p_{N}^{A F}$ is expressed as in (35).

Proof of Lemmo2 2. Using standard upper bound $e^{x} \mathcal{E}_{1}(x) \approx$ $\frac{1}{1+x}[35,5.1 .19]$ into $(75)$ and $(76)$, and using some algebraic simplifications we obtain

$$
\begin{aligned}
& I_{6} \approx \frac{\lambda_{z} \vartheta_{2} e^{-\lambda_{z} \xi_{1}}\left(e^{-\lambda_{z} \chi_{N}}-e^{-\lambda_{z} \chi_{F}}\right)}{1+\lambda_{z}\left(\chi_{N}+\xi_{1}+\vartheta_{2}\right)} \\
&-e^{\xi_{1}\left(C_{\ell}+\lambda_{z}-1\right)}\left(e^{\left.-\chi_{N}-e^{-\chi_{F}}\right),}\right. \\
& \text { and } I_{7} \approx e^{\xi_{1}\left(\lambda_{z}-C_{\ell}\right)}+\frac{\lambda_{z} \vartheta_{2} e^{\xi_{1}\left(C_{\ell}-\lambda_{z}\right)}}{1+\lambda_{z}\left(\vartheta_{2}+2 \xi_{1}\right)}
\end{aligned}
$$

Note that the derived expressions for $I_{3}, I_{4}$ and $I_{5}$ in (77), 73) and (74) are already simplified. Therefore, substituting $I_{3}$ to $I_{7}$ into (32), we obtain the high-SNR approximated expression for $p_{N}^{A F}$ as in 36.

\section{APPENDIX D}

Proof of Theorem 4: Using (6), (12), (13) and (16) into 39, $\operatorname{Pr}\left\{A_{1}\right\}$ can be expressed as

$$
\begin{aligned}
\operatorname{Pr}\left\{A_{1}\right\}= & \operatorname{Pr}\left\{\frac{\left(1-\alpha_{B}\right) \rho_{B} X}{\alpha_{B} \rho_{B} X+1} \geq \gamma_{F}, \alpha_{B} \rho_{B} X \geq \gamma_{N},\right. \\
& \left.\frac{\left(1-\alpha_{R}\right) \rho_{R} Y}{\alpha_{R} \rho_{R} Y+1}+\frac{\left(1-\alpha_{B}\right) \rho_{B} W}{\alpha_{B} \rho_{B} W+1} \geq \gamma_{F}\right\} \\
= & \operatorname{Pr}\left\{X \geq \max \left(\chi_{F}, \chi_{N}\right)\right\}\left[\operatorname{Pr}\left\{W \geq \chi_{F}\right\}\right. \\
+ & \operatorname{Pr}\left\{\chi_{F}>W>\max \left(0, \frac{-\phi_{R}}{\rho_{B}\left(\alpha_{B}\left(1-\alpha_{R}\right)+\alpha_{R} \phi\right)}\right)\right. \\
& \left.\left.\times Y \geq \frac{\left(\gamma_{F}-\phi \rho_{B} W\right)}{\rho_{R}\left(\phi_{R}+\left(1-\alpha_{R}\right) \alpha_{B} \rho_{B} W+\alpha_{R} \phi \rho_{B} W\right)}\right\}\right] .(80)
\end{aligned}
$$

Since the RVs $W, X$ and $Y$ are independent and have an exponential distribution, we obtain

$$
\begin{aligned}
\operatorname{Pr}\left\{A_{1}\right\} & =e^{-\lambda_{x} \max \left(\chi_{F}, \chi_{N}\right)}\left[e^{-\lambda_{w} \chi_{F}}\right. \\
& \left.+\int_{\max \left(0, \frac{\phi_{R}}{\vartheta_{1}}\right)}^{\chi_{F}} \lambda_{w} \exp \left(\frac{\lambda_{y} \phi \rho_{B}\left(w-\chi_{F}\right)}{\rho_{R} \vartheta_{1}\left(w+\frac{\phi_{R}}{\vartheta_{1}}\right)}-\lambda_{w} w\right) d w\right] .
\end{aligned}
$$

After substituting $\lambda_{w}\left(w+\frac{\phi_{R}}{\vartheta_{1}}\right)=w$ and solving, we obtain

$$
\begin{aligned}
\operatorname{Pr}\left\{A_{1}\right\}= & e^{-\lambda_{x} \max \left(\chi_{F}, \chi_{N}\right)}\left[e^{-\lambda_{w} \chi_{F}}+\exp \left(\frac{\lambda_{w} \phi_{R}}{\vartheta_{1}}+\frac{\lambda_{y} \phi \rho_{B}}{\rho_{R} \vartheta_{1}}\right)\right. \\
& \left.\times \int_{\max \left(\frac{\lambda_{w \phi_{R}}}{\vartheta_{1}}, 0\right)} \exp \left(\frac{-\lambda_{y} \lambda_{w} \phi \rho_{B}\left(\frac{\phi_{R}}{\vartheta_{1}}+\chi_{F}\right)}{\rho_{R} \vartheta_{1} w}-w\right) d w\right] .(82)
\end{aligned}
$$

Using an approach similar to that used to derive [58, we obtain

$$
\begin{aligned}
\operatorname{Pr}\left\{A_{1}\right\} & =e^{-\lambda_{x} \max \left(\chi_{F}, \chi_{N}\right)}\left[e^{-\lambda_{w} \chi_{F}}+\exp \left(\frac{\lambda_{w} \phi_{R}}{\vartheta_{1}}+\frac{\lambda_{y} \phi \rho_{B}}{\rho_{R} \vartheta_{1}}\right)\right. \\
& \times\left\{\Gamma\left(1, \max \left(\frac{\lambda_{w} \phi_{R}}{\vartheta_{1}}, 0\right) ; \frac{-\lambda_{y} \lambda_{w} \phi \rho_{B}\left(\frac{\phi_{R}}{\vartheta_{1}}+\chi_{F}\right)}{\rho_{R} \vartheta_{1}}\right)\right. \\
& \left.\left.-\Gamma\left(1, \lambda_{w}\left(\chi_{F}+\frac{\phi_{R}}{\vartheta_{1}}\right) ; \frac{-\lambda_{y} \lambda_{w} \phi \rho_{B}\left(\frac{\phi_{R}}{\vartheta_{1}}+\chi_{F}\right)}{\rho_{R} \vartheta_{1}}\right)\right\}\right]
\end{aligned}
$$

Similarly, the expression for $\operatorname{Pr}\left\{A_{2}\right\}$ is obtained as

$$
\begin{aligned}
\operatorname{Pr}\left\{A_{2}\right\}= & \left(e^{-\lambda_{x} \chi_{F}}-e^{-\lambda_{x} \chi_{N}}\right)\left[e^{-\frac{\lambda_{y} \gamma_{F}}{\rho_{R}}}+\exp \left(\frac{\lambda_{w}}{\alpha_{B} \rho_{B}}+\frac{\lambda_{y} \phi}{\alpha_{B} \rho_{R}}\right)\right. \\
& \times\left\{\Gamma\left(1, \frac{\lambda_{y} \phi}{\alpha_{B} \rho_{R}} ; \frac{\lambda_{y} \lambda_{w}\left(\phi+\alpha_{B} \gamma_{F}\right)}{\alpha_{B}^{2} \rho_{B} \rho_{R}}\right)\right. \\
& \left.-\Gamma\left(1, \frac{\lambda_{y}}{\rho_{R}}\left(\gamma_{F}+\frac{\phi}{\alpha_{B}}\right) ; \frac{\lambda_{y} \lambda_{w}\left(\phi+\alpha_{B} \gamma_{F}\right)}{\alpha_{B}^{2} \rho_{B} \rho_{R}}\right)\right\} .
\end{aligned}
$$

Further, using (6), (12) and (13), $\operatorname{Pr}\left\{A_{3}\right\}$ of (39) can be expressed as

$$
\operatorname{Pr}\left\{A_{3}\right\}=e^{-\lambda_{w} \chi_{F}}\left(1-e^{-\lambda_{x} \chi_{F}}\right) .
$$

Using (83), 84) and 85) in (39), $p_{F}^{D F}$ is obtained as in 40).

\section{APPENDIX E}

Proof of Lemma 4. For higher values of $\rho_{B}$ and $\rho_{R}$, we use $e^{-\frac{a}{x}}=\frac{1}{1+\frac{a}{x}}$ in 82 to obtain

$$
\begin{gathered}
\operatorname{Pr}\left\{A_{1}\right\} \simeq e^{-\lambda_{x} \max \left(\chi_{F}, \chi_{N}\right)}\left[e^{-\lambda_{w} \chi_{F}}+\exp \left(\frac{\lambda_{w} \phi_{R}}{\vartheta_{1}}+\frac{\lambda_{y} \phi \rho_{B}}{\rho_{R} \vartheta_{1}}\right)\right. \\
\left.\times \int_{\max \left(\frac{\lambda_{w} \phi_{R}}{\vartheta_{1}}, 0\right)}^{\lambda_{w}\left(\chi_{F}+\frac{\phi_{R}}{\vartheta_{1}}\right)}\left(1-\frac{\kappa}{w+\kappa}\right) e^{-w} d w\right],
\end{gathered}
$$

where $\left.\kappa=\frac{\lambda_{y} \lambda_{w} \phi \rho_{B}}{3 \vartheta_{1}}+\chi_{F}\right)$. Solving the above integral using [34, 3.352z.3], we obtain

$\operatorname{Pr}\left\{A_{1}\right\} \simeq e^{-\lambda_{x} \max \left(\chi_{F}, \chi_{N}\right)}\left[\exp \left(\frac{\lambda_{w} \phi_{R}}{\vartheta_{1}}+\frac{\lambda_{y} \phi \rho_{B}}{\rho_{R} \vartheta_{1}}\right)\{\exp (-\max \right.$

$$
\begin{aligned}
& \left.\left(\frac{\lambda_{w} \phi_{R}}{\vartheta_{1}}, 0\right)\right) c-\exp \left(-\lambda_{w}\left(\chi_{F}+\frac{\phi_{R}}{\vartheta_{1}}\right)\right)-\kappa e^{\kappa}\left[\mathcal{E}_{1}(\max \right. \\
& \left.\left.\left.\left.\left(\frac{\lambda_{w} \phi_{R}}{\vartheta_{1}}, 0\right)+\kappa\right)-\mathcal{E}_{1}\left(\lambda_{w}\left(\chi_{F}+\frac{\phi_{R}}{\vartheta_{1}}\right)+\kappa\right)\right]\right\}+e^{-\lambda_{w} \chi_{F}}\right] .(87)
\end{aligned}
$$

At high-SNRs, $\kappa \propto \frac{1}{\rho_{B}^{2}}$. We therefore neglect the product term of $\kappa e^{\kappa}$. Now $\operatorname{Pr}\left\{A_{1}\right\}$ is expressed as

$$
\begin{aligned}
\operatorname{Pr}\left\{A_{1}\right\} \simeq & e^{-\lambda_{x} \max \left(\chi_{F}, \chi_{N}\right)}\left[\exp \left(\frac{\lambda_{w} \phi_{R}}{\vartheta_{1}}+\frac{\lambda_{y} \phi \rho_{B}}{\rho_{R} \vartheta_{1}}-\max \left(\frac{\lambda_{w} \phi_{R}}{\vartheta_{1}}, 0\right)\right)\right. \\
& \left.+e^{-\lambda_{w} \chi_{F}}\left(1-\exp \left(\frac{\lambda_{y} \phi \rho_{B}}{\rho_{R} \vartheta_{1}}\right)\right)\right] .
\end{aligned}
$$

Similarly, solving for $A_{2}$, we obtain

$$
\begin{aligned}
\operatorname{Pr}\left\{A_{2}\right\} \simeq & \left(e^{-\lambda_{x} \chi_{F}}-e^{-\lambda_{x} \chi_{N}}\right)\left[\exp \left(\frac{\lambda_{w}}{\alpha_{B} \rho_{B}}\right)+\exp \left(-\frac{\lambda_{y} \gamma_{F}}{\rho_{R}}\right)\right. \\
& \left.\left(1-\exp \left(\frac{\lambda_{w}}{\alpha_{B} \rho_{B}}\right)\right)\right] .
\end{aligned}
$$


Substituting for $\operatorname{Pr}\left\{A_{1}\right\}$ and $\operatorname{Pr}\left\{A_{2}\right\}$ from 87 and 89 into (39), we obtain $p_{F}^{D F}$ given by (41).

\section{APPENDIX F}

Proof of Theorem 5. Using (4), (5), (12) and (13) in (45), $\operatorname{Pr}\left\{B_{1}\right\}$ can be expressed as

$$
\begin{aligned}
\operatorname{Pr}\left\{B_{1}\right\} & =\operatorname{Pr}\left\{\Gamma_{R F}^{I}<\gamma_{F}, \Gamma_{N F}^{I} \geq \gamma_{F}, \Gamma_{N N}^{I} \geq \gamma_{N}\right\} \\
& =\operatorname{Pr}\left\{\frac{\left(1-\alpha_{B}\right) \rho_{B} X}{\alpha_{B} \rho_{B} X+1}<\gamma_{F}, \frac{\left(1-\alpha_{B}\right) \rho_{B} Z}{\alpha_{B} \rho_{B} Z+1} \geq \gamma_{F}, \alpha_{B} \rho_{B} Z \geq \gamma_{N}\right\} \\
& =\operatorname{Pr}\left\{X<\chi_{F}, Z \geq \max \left(\chi_{F}, \chi_{N}\right)\right\} U(\phi) \\
& =e^{-\lambda_{z} \max \left(\chi_{F}, \chi_{N}\right)}\left[1-e^{-\lambda_{x} \chi_{F}}\right] U(\phi)
\end{aligned}
$$

Using (45, $\operatorname{Pr}\left\{B_{2}\right\}$ is given by

$$
\begin{aligned}
\operatorname{Pr}\left\{B_{2}\right\} & =\operatorname{Pr}\left\{\Gamma_{R F}^{I} \geq \gamma_{F}, \Gamma_{R N}^{I}<\gamma_{N}, \Gamma_{N F-F}^{C O M} \geq \gamma_{F}, \Gamma_{N N}^{I} \geq \gamma_{N}\right\} \\
& =\operatorname{Pr}\left\{\chi_{F}<X<\chi_{N}, \rho_{B} Z(\underbrace{\left(1-\alpha_{B}\right)-\alpha_{B}\left(\gamma_{F}-\rho_{R} V\right)}_{\left(\phi+\alpha_{B} \rho_{R} V\right)})\right. \\
& \left.>\left(\gamma_{F}-\rho_{R} V\right), Z \geq \chi_{N}\right\} U(\phi) \\
& =\operatorname{Pr}\left\{\chi_{F}<X<\chi_{N}, V>\frac{\gamma_{F}}{\rho_{R}}, Z \geq \chi_{N}\right\}+\operatorname{Pr}\left\{\chi_{F}<X\right. \\
& \left.<\chi_{N}, V<\frac{\gamma_{F}}{\rho_{R}}, Z \geq \max \left(\chi_{N}, \frac{\gamma_{F}-\rho_{R} V}{\phi+\alpha_{B} \phi_{R} V}\right)\right\} .
\end{aligned}
$$

To satisfy $\chi_{N}>\chi_{F}, \max \left(\chi_{N}, \frac{\gamma_{F}-\rho_{R} V}{\phi+\alpha_{B} \phi_{R} V}\right)=\chi_{N}$. Using this fact, the above can be simplified as

$$
\begin{aligned}
\operatorname{Pr}\left\{B_{2}\right\} & =\operatorname{Pr}\left\{\chi_{F}<X<\chi_{N}, Z \geq \chi_{N}\right\} \\
& =e^{-\lambda_{z} \chi_{N}}\left(e^{-\lambda_{x} \chi_{F}}-e^{-\lambda_{x} \chi_{N}}\right) U\left(\phi-\frac{\alpha_{B} \gamma_{F}}{\gamma_{N}}\right) .
\end{aligned}
$$

Further, the expression for $\operatorname{Pr}\left\{B_{3}\right\}$ is calculated as

$$
\begin{aligned}
\operatorname{Pr}\left\{B_{3}\right\} & =\operatorname{Pr}\left\{\Gamma_{R F}^{I} \geq \gamma_{F}, \Gamma_{R N}^{I} \geq \gamma_{N}, \Gamma_{N F-S}^{C O M} \geq \gamma_{F}, \Gamma_{N N-S}^{C O M} \geq \gamma_{N}\right\} \\
& =\operatorname{Pr}\left\{X>\max \left(\chi_{F}, \chi_{N}\right), \rho_{R} V\left(\left(1-\alpha_{R}\right)\left(1+\alpha_{B} \rho_{B} Z\right)-\right.\right. \\
& \left.\left.\alpha_{R}\left(\gamma_{F}-\phi \rho_{B} Z\right)\right)>\left(\gamma_{F}-\phi \rho_{B} Z\right), V \geq \frac{\gamma_{N}-\alpha_{B} \rho_{B} Z}{\alpha_{R} \rho_{B}}, z<\chi_{N}\right\} .
\end{aligned}
$$

Using the above, the conditions for outage on ranges of $X$, $V$ and $Z$ with $\phi>0$ and $\phi<0$ are listed in Table IV. Now, $\operatorname{Pr}\left\{B_{3}\right\}$ can be expressed as

$$
\begin{aligned}
\operatorname{Pr}\left\{B_{3}\right\} & =\left[\operatorname{Pr}\left\{Z>\max \left(\chi_{F}, \chi_{N}\right)\right\}+\operatorname{Pr}\left\{\chi_{F}<z<\chi_{N}, V\right.\right. \\
& \left.\geq \tilde{\chi_{N_{1}}}(z)\right\}+\operatorname{Pr}\left\{\chi_{F}>Z>\max \left(\chi_{N}, \vartheta_{5}\right), V \geq \tilde{\chi_{N_{1}}}\right\} \\
& +\operatorname{Pr}\left\{V \geq \tilde{\chi_{N_{1}}}(Z), \min \left(\chi_{F}, \chi_{N}\right)>Z>\max \left(\vartheta_{5}, \vartheta_{4}\right)\right\} \\
& +\operatorname{Pr}\left\{\min \left(\chi_{F}, \chi_{N}, \vartheta_{4}\right)>Z>\max \left(0, \vartheta_{5}\right),\right. \\
& \left.\left.V \geq \tilde{\chi_{N_{2}}}(Z)\right\}\right] \operatorname{Pr}\left\{X>\max \left(\chi_{F}, \chi_{N}\right)\right\}
\end{aligned}
$$

where $\tilde{\chi_{N_{1}}}(Z)=\frac{\gamma_{F}-\phi \rho_{B} Z}{\rho_{R}\left(\phi_{R}+\rho_{B} Z\left(\phi \alpha_{R}+\alpha_{B}\left(1-\alpha_{R}\right)\right)\right)}$ and $\tilde{\chi_{N_{2}}}(Z)=$ $\frac{\gamma_{N}-\alpha_{B} \rho_{B} Z}{\alpha_{R} \rho_{B}}$. After averaging over the PDFs of RVs $V$,
$X$ and $Z$, we obtain $\operatorname{Pr}\left\{B_{3}\right\}$ as in (95), where $\omega=$ $\frac{-\lambda_{v}\left(\gamma_{F}-\phi \rho_{B} z\right)}{\rho_{R}\left(\phi_{R}+\rho_{B} z\left(\phi \alpha_{R}+\alpha_{B}\left(1-\alpha_{R}\right)\right)\right)}, \tilde{\chi_{N}}=\min \left(\chi_{F}, \chi_{N}, \vartheta_{4}\right)$. After some mathematical rearrangement, the above can be expressed as

$$
\begin{aligned}
\operatorname{Pr}\left\{B_{3}\right\}= & e^{-\lambda_{x} \max \left(\chi_{F}, \chi_{N}\right)}\left[e^{-\lambda_{z} \max \left(\chi_{F}, \chi_{N}\right)}+\frac{\lambda_{z} e^{-\varepsilon_{3} \gamma_{N}}}{\varepsilon_{3} \alpha_{B} \rho_{B}-\lambda_{z}} \times\right. \\
& {\left[\exp \left(\varepsilon_{3} \alpha_{B} \rho_{B} \chi_{N}-\lambda_{z} \chi_{N}\right)-\exp \left(\varepsilon_{3} \alpha_{B} \rho_{B} \chi_{F}-\lambda_{z} \chi_{F}\right)\right] } \\
& +\frac{\lambda_{z} e^{-\varepsilon_{3} \gamma_{N}}}{\varepsilon_{3} \alpha_{B} \rho_{B}-\lambda_{z}}\left[\exp \left(\varepsilon_{3} \alpha_{B} \rho_{B} \tilde{\chi}_{N}-\lambda_{z} \tilde{\chi}_{N}\right)-\exp \left(\varepsilon_{3} \alpha_{B} \rho_{B}\right.\right. \\
& \left.\left.\max \left(0, \frac{-\phi_{R}}{\vartheta_{1}}\right)-\lambda_{z} \max \left(0, \frac{-\phi_{R}}{\vartheta_{1}}\right)\right)\right] \\
& +\underbrace{\int_{F}^{\max \left(\chi_{N}, \frac{-\phi_{R}}{\vartheta_{1}}\right)} \exp \left(\frac{\lambda_{v} \phi \rho_{B}}{\rho_{R} \vartheta} \times \frac{z-\chi_{F}}{z+\frac{\phi_{R}}{\vartheta_{1}}}\right) \lambda_{z} e^{-\lambda_{z} z} d z}_{I_{8}} \\
& +\int_{\left.\int_{-\phi_{R}}^{\min \left(\chi_{F}, \chi_{N}\right)} \exp \left(\frac{\lambda_{v} \phi \rho_{B}}{\rho_{R} \vartheta_{1}} \times \frac{z-\chi_{F}}{z+\frac{\phi_{R}}{\vartheta_{1}}}\right) \lambda_{z} e^{-\lambda_{z} z} d z\right]}^{\max (96)}
\end{aligned}
$$

where $\varepsilon_{3}=\frac{\lambda_{v}}{\alpha_{R} \rho_{R}}$. Substituting $\lambda_{z}\left(z+\frac{\phi_{R}}{\vartheta_{1}}\right)=z$, and using 136 , eq. (4)], $I_{8}$ and $I_{9}$ are obtained as

$$
\begin{aligned}
I_{8}= & \exp \left(\frac{\phi_{R} \lambda_{z} \rho_{R}+\lambda_{v} \phi \rho_{B}}{\rho_{R} \vartheta_{1}}\right)\left[\Gamma\left(1, \lambda_{z} \max \left(0, \chi_{N}+\frac{\phi_{R}}{\vartheta_{1}}\right) ; \tilde{\chi_{F}}\right)\right. \\
& \left.-\Gamma\left(1, \lambda_{z}\left(\chi_{F}+\frac{\phi_{R}}{\vartheta_{1}}\right) ; \tilde{\chi_{F}}\right)\right], \\
I_{9}= & \exp \left(\frac{\phi_{R} \lambda_{z} \rho_{R}+\lambda_{v} \phi \rho_{B}}{\rho_{R} \vartheta_{1}}\right)\left[\Gamma\left(1, \lambda_{z} \max \left(0, \vartheta_{4}+\frac{\phi_{R}}{\vartheta_{1}}\right) ; \tilde{\chi_{F}}\right)\right. \\
& \left.-\Gamma\left(1, \lambda_{z} \min \left(\chi_{N}, \chi_{F}\right)+\frac{\phi_{R} \lambda_{z}}{\vartheta_{1}} ; \tilde{\chi_{F}}\right)\right],
\end{aligned}
$$

where $\tilde{\chi_{F}}=\frac{\lambda_{z} \lambda_{v} \phi \rho_{B}\left(\chi_{F}+\frac{\phi_{R}}{\vartheta_{1}}\right)}{\vartheta_{1}}$. Using $I_{8}$ and $I_{9}$ from 97) and 98 into $96, \stackrel{\operatorname{Pr}}{\operatorname{Pr}}\left\{B_{3}\right\}$ is expressed as

$$
\begin{aligned}
& \operatorname{Pr}\left\{B_{3}\right\}=e^{-\lambda_{x} \max \left(\chi_{F}, \chi_{N}\right)}\left[e^{-\lambda_{z} \max \left(\chi_{F}, \chi_{N}\right)}+\frac{\lambda_{z} e^{-\varepsilon_{3} \gamma_{N}}}{\varepsilon_{3} \alpha_{B} \rho_{B}-\lambda_{z}}\right. \\
& \times\left[\exp \left(\varepsilon_{3} \alpha_{B} \rho_{B} \chi_{N}-\lambda_{z} \chi_{N}\right)-\exp \left(\varepsilon_{3} \alpha_{B} \rho_{B} \chi_{F}-\lambda_{z} \chi_{F}\right)\right. \\
& \left.+\frac{\exp \left(\varepsilon_{3} \alpha_{B} \rho_{B} \tilde{\chi_{N}}\right)}{\exp \left(\tilde{\chi_{N}} \lambda_{z}\right)}-\frac{\exp \left(\varepsilon_{3} \alpha_{B} \rho_{B} \max \left(0, \frac{-\phi_{R}}{\vartheta_{1}}\right)\right)}{\exp \left(\max \left(0, \frac{-\phi_{R}}{\vartheta_{1}}\right) \lambda_{z}\right)}\right] \\
& +\exp \left(\frac{\phi_{R} \lambda_{z} \rho_{R}+\lambda_{\nu} \phi \rho_{B}}{\rho_{R} \vartheta_{1}}\right)\left[\Gamma\left(1, \lambda_{z} \max \left(0, \chi_{N}+\frac{\phi_{R}}{\vartheta_{1}}\right) ; \tilde{\chi_{F}}\right)\right. \\
& -\Gamma\left(1, \lambda_{z}\left(\chi_{F}+\frac{\phi_{R}}{\vartheta_{1}}\right) ; \tilde{\chi_{F}}\right)+\Gamma\left(1, \lambda_{z} \max \left(0, \vartheta_{4}+\frac{\phi_{R}}{\vartheta_{1}}\right) ; \tilde{\chi_{F}}\right) \\
& \left.\left.-\Gamma\left(1, \lambda_{z} \min \left(\chi_{N}, \chi_{F}\right)+\frac{\phi_{R} \lambda_{z}}{\vartheta_{1}} ; \tilde{\chi_{F}}\right)\right]\right]
\end{aligned}
$$

Substituting (90), 92 and 99 into 45, $p_{N}^{D F}$ is obtained as in (46). 
TABLE IV: $B_{3}$ - ranges of $V, X$ and $Z$ for different values of $\alpha_{B}$

\begin{tabular}{|c|c|c|}
\hline \multirow{4}{*}{$\alpha_{B}<\frac{1}{1+\gamma_{F}}$} & $Z>\max \left(\chi_{F}, \chi_{N}\right)$ & $V>0, X>\max \left(\chi_{F}, \chi_{N}\right)$ \\
\cline { 2 - 3 } & $\chi_{N}>Z>\chi_{F}$ & $V>\tilde{\chi_{N_{2}}}(Z), X>\max \left(\chi_{F}, \chi_{N}\right)$ \\
\cline { 2 - 3 } & $\chi_{F}>Z>\max \left(\chi_{N}, \vartheta_{5}\right)$ & $V>\tilde{\chi_{N_{1}}}(Z), X>\max \left(\chi_{F}, \chi_{N}\right)$ \\
\cline { 2 - 3 } & $\begin{array}{c}\min \left(\chi_{F}, \chi_{N}\right)>Z>\vartheta_{5} \\
\alpha_{B} \geq \frac{1}{1+\gamma_{F}}\end{array}$ & $\begin{array}{c}V>\max \left(\tilde{\chi_{N}}(Z), \tilde{\chi_{N}}(Z)\right), X>\max \left(\chi_{F}, \chi_{N}\right) \\
\text { if } \tilde{\chi_{N_{1}}}(Z) \gtrless \tilde{\chi_{N_{2}}}(Z) \Rightarrow Z \gtrless \vartheta_{4}\end{array}$ \\
\hline
\end{tabular}

$$
\begin{aligned}
\operatorname{Pr}\left\{B_{3}\right\} & =e^{-\lambda_{x} \max \left(\chi_{F}, \chi_{N}\right)}\left[e^{-\lambda_{z} \max \left(\chi_{F}, \chi_{N}\right)}+\int_{\chi_{F}}^{\chi_{N}} \exp \left(\frac{-\lambda_{v}\left(\gamma_{N}-\alpha_{B} \rho_{B} z\right)}{\alpha_{R} \rho_{R}}\right) \lambda_{z} e^{-\lambda_{z} z} d z+\int_{\max \left(0, \frac{-\phi_{R}}{\vartheta_{1}}\right)}^{\chi_{N}} \exp \left(\frac{-\lambda_{v}\left(\gamma_{N}-\alpha_{B} \rho_{B} z\right)}{\alpha_{R} \rho_{R}}\right)\right. \\
& \left.\times \lambda_{z} e^{-\lambda_{z} z} d z+\int_{\max \left(\chi_{N}, \frac{-\phi_{R}}{\vartheta_{1}}\right)}^{\chi_{F}} \exp \left(\frac{-\lambda_{v}\left(\gamma_{F}-\phi \rho_{B} z\right)}{\rho_{R}\left(\phi_{R}+\rho_{B} z \omega\right)}\right) \lambda_{z} e^{-\lambda_{z} z} d z+\int_{\max \left(\frac{\phi_{F}}{\vartheta_{1}}, \vartheta_{4}\right)}^{\min \left(\chi_{N}\right)} \exp \left(\frac{-\lambda_{v}\left(\gamma_{F}-\phi \rho_{B} z\right)}{\rho_{R}\left(\phi_{R}+\rho_{B} z \omega\right)}\right) \lambda_{z} e^{-\lambda_{z} z} d z\right],
\end{aligned}
$$

\section{REFERENCES}

[1] Y. Liu, Z. Qin, M. Elkashlan, Z. Ding, A. Nallanathan, and L. Hanzo, "Nonorthogonal multiple access for 5G and beyond," Proc. IEEE, vol. 105, no. 12, pp. 2347-2381, 2017.

[2] L. Dai, B. Wang, Y. Yuan, S. Han, I. Chih-lin, and Z. Wang, "Nonorthogonal multiple access for 5G: solutions, challenges, opportunities, and future research trends," IEEE Cummun. Mag., vol. 53, no. 9 , pp. 74-81, 2015.

[3] M. Vaezi, Z. Ding, and H. V. Poor, Multiple Access Techniques for $5 G$ Wireless Networks and Beyond. Springer, 2019.

[4] O. Maraqa, A. S. Rajasekaran, S. Al-Ahmadi, H. Yanikomeroglu, and S. M. Sait, "A survey of rate-optimal power domain NOMA with enabling technologies of future wireless networks," IEEE Commun. Surv. Tuts., vol. 22, no. 4, pp. 2192-2235, 2020.

[5] Z. Ding, X. Lei, G. K. Karagiannidis, R. Schober, J. Yuan, and V. K. Bhargava, "A survey on non-orthogonal multiple access for 5G networks: Research challenges and future trends," IEEE J. Sel. Areas Commun., vol. 35, no. 10, pp. 2181-2195, 2017.

[6] W. Shin, M. Vaezi, B. Lee, D. J. Love, J. Lee, and H. V. Poor, "Non-orthogonal multiple access in multi-cell networks: Theory, performance, and practical challenges," IEEE Commun. Mag., vol. 55, no. 10, pp. 176-183, 2017.

[7] Z. Ding, Y. Liu, J. Choi, Q. Sun, M. Elkashlan, I. Chih-Lin, and H. V. Poor, "Application of non-orthogonal multiple access in LTE and 5G networks," IEEE Commun. Mag., vol. 55, no. 2, pp. 185-191, 2017.

[8] Z. Yang, Z. Ding, P. Fan, and N. Al-Dhahir, "A general power allocation scheme to guarantee quality of service in downlink and uplink NOMA systems," IEEE Trans. Wireless Commun., vol. 15, no. 11, pp. 7244-7257, Nov 2016.

[9] Y. Xiao, L. Hao, Z. Ma, Z. Ding, Z. Zhang, and P. Fan, "Forwarding strategy selection in dual-hop NOMA relaying systems," IEEE Commun. Lett., vol. 22, no. 8, pp. 1644-1647, 2018.

[10] Z. Yang, Z. Ding, Y. Wu, and P. Fan, "Novel relay selection strategies for cooperative NOMA," IEEE Trans. Veh. Technol., vol. 66, no. 11, pp. 10114-10 123, 2017.

[11] Z. Ding, H. Dai, and H. V. Poor, "Relay selection for cooperative NOMA," IEEE Wireless Commun. Lett., vol. 5, no. 4, pp. 416-419, 2016.

[12] K. Agrawal, M. F. Flanagan, and S. Prakriya, "NOMA with batteryassisted energy harvesting full-duplex relay," IEEE Trans. Veh. Technol., vol. 69, no. 11, pp. 13952-13957, 2020.

[13] F. Kara and H. Kaya, "Improved user fairness in decode-forward relaying non-orthogonal multiple access schemes with imperfect sic and csi," IEEE Access, vol. 8, pp. 97540-97 556, 2020.

[14] L. Han, W.-P. Zhu, and M. Lin, "Outage of NOMA-based hybrid satellite-terrestrial multi-antenna DF relay networks," IEEE Wireless Commun. Lett., vol. 10, no. 5, pp. 1083-1087, 2021.
[15] D. Wan, M. Wen, F. Ji, Y. Liu, and Y. Huang, "Cooperative NOMA systems with partial channel state information over nakagami- $m$ fading channels," IEEE Trans. Commun., vol. 66, no. 3, pp. 947-958, 2018.

[16] X. Yue, Y. Liu, S. Kang, A. Nallanathan, and Z. Ding, "Exploiting full/half-duplex user relaying in NOMA systems," IEEE Trans. Commun., vol. 66, no. 2, pp. 560-575, 2018.

[17] K. Janghel and S. Prakriya, "Performance of adaptive OMA/cooperative-NOMA scheme with user selection," IEEE Commun. Lett., vol. 22, no. 10, pp. 2092-2095, Oct 2018.

[18] K. Janghel, A. Jee, and S. Prakriya, "Performance of underlay cooperative hybrid OMA/NOMA scheme with user selection," in IEEE PIMRC, 2020, pp. 1-6.

[19] X. Lai, Q. Zhang, and J. Qin, "Cooperative NOMA short-packet communications in flat rayleigh fading channels," IEEE Trans. Veh. Technol., vol. 68, no. 6, pp. 6182-6186, 2019.

[20] Q. Y. Liau and C. Y. Leow, "Successive user relaying in cooperative NOMA system," IEEE Wireless Commun. Lett., vol. 8, no. 3, pp. 921-924, 2019.

[21] T. T. Nguyen, T. V. Nguyen, T. H. Vu, D. B. d. Costa, and C. D. Ho, "IoT-based coordinated direct and relay transmission with nonorthogonal multiple access," IEEE Wireless Cummun. Letters, vol. 10 , no. 3, pp. 503-507, 2021.

[22] Y. Xu, B. Li, N. Zhao, Y. Chen, G. Wang, Z. Ding, and X. Wang, "Coordinated direct and relay transmission with NOMA and network coding in nakagami-m fading channels," IEEE Trans. Cummun., vol. 69 , no. 1, pp. 207-222, 2021.

[23] H. Liu, N. I. Miridakis, T. A. Tsiftsis, K. J. Kim, and K. S. Kwak, "Coordinated uplink transmission for cooperative NOMA systems," in 2018 IEEE Global Commun. Conf. (GLOBECOM), 2018, pp. 1-6.

[24] Y. Xu, J. Cheng, G. Wang, and V. C. M. Leung, "Coordinated direct and relay transmission for multiuser networks: NOMA or hybrid multiple access?" IEEE Wireless Communic. Lett., vol. 10, no. 5, pp. 976-980, 2021.

[25] Y. Xu, J. Cheng, G. Wang, and V. C. Leung, "Adaptive coordinated direct and relay transmission for NOMA networks: A joint downlinkuplink scheme," IEEE Trans. Wireless Commun., pp. 1-1, 2021.

[26] H. Liu, Z. Ding, K. J. Kim, K. S. Kwak, and H. V. Poor, "Decode-andforward relaying for cooperative NOMA systems with direct links," IEEE Trans. Wireless Commun., vol. 17, no. 12, pp. 8077-8093, 2018.

[27] X. Pei, H. Yu, M. Wen, S. Mumtaz, S. Al Otaibi, and M. Guizani, "NOMA-based coordinated direct and relay transmission with a halfduplex/ full-duplex relay," IEEE Trans. Commun., vol. 68, no. 11, pp. 6750-6760, 2020.

[28] X. Yue, Y. Liu, S. Kang, and A. Nallanathan, "Performance analysis of NOMA with fixed gain relaying over nakagami- $m$ fading channels," IEEE Access, vol. 5, pp. 5445-5454, 2017. 
[29] J. Kim and I. Lee, "Non-orthogonal multiple access in coordinated direct and relay transmission," IEEE Commun. Lett., vol. 19, no. 11, pp. 2037-2040, 2015.

[30] K. Cao, B. Wang, H. Ding, T. Li, and F. Gong, "Optimal relay selection for secure NOMA systems under untrusted users," IEEE Trans. Veh. Technol., vol. 69, no. 2, pp. 1942-1955, 2020.

[31] X. Li, J. Li, Y. Liu, Z. Ding, and A. Nallanathan, "Residual transceiver hardware impairments on cooperative NOMA networks," IEEE Trans. Wireless Cummun., vol. 19, no. 1, pp. 680-695, 2020.

[32] M. Vaezi, R. Schober, Z. Ding, and H. V. Poor, "Non-orthogonal multiple access: Common myths and critical questions," IEEE Wireless Commun., vol. 26, no. 5, pp. 174-180, 2019.

[33] Z. Ding, R. Schober, and H. V. Poor, "Unveiling the importance of SIC in NOMA systems - part 1: State of the art and recent findings," IEEE Commun. Lett., vol. 24, no. 11, pp. 2373-2377, 2020.

[34] I. S. Gradshteyn and I. M. Ryzhik, Table of Integrals, Series, and Products, 7th ed. San Diego, CA, USA: Academic, 2007.

[35] M. Abramowitz and I. A. Stegun, Handbook of Mathematical Functions: with Formulas, Graphs, and Mathematical Tables. New York, NY, USA: Dover, 1965.

[36] M. Chaudhry and S. Zubair, "Generalized incomplete gamma functions with applications," Journal of Computational and Applied Mathematics, vol. 55, no. 1, pp. 99 - 123, 1994.

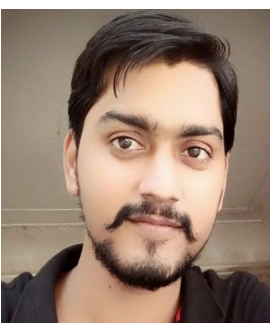

Anand Jee (Graduate Student Member, IEEE) received the B.Tech. degree with Distinction in electronics and communication engineering from The ICFAI University Dehradun, Uttrakhand, India, and the M.Tech degree in communication and signal processing Engineering (CSPE) from the National Institute of Technology (NIT) Silchar, Assam, India. He secured fourth rank in B.tech and received a postgraduate silver medal for securing the highest cumulative performance index CPI) in M.Tech. He is currently pursuing the $\mathrm{Ph} . \mathrm{D}$. degree from the department of electrical engineering, Indian Institute of Technology (IIT) Delhi, New Delhi, India. His research interests include non-orthogonal multiple access (NOMA), cognitive radios, cooperative communications, energy harvesting, Intelligent reflecting surfaces and backscatter communication.

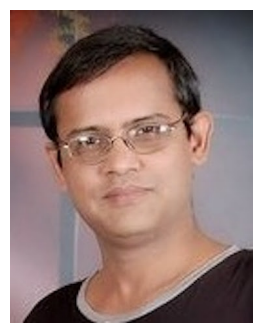

Shankar Prakriya (SM'02) received the B.E. degree (Hons) in electronics and communication engineering from the Regional Engineering College, Bharathidasan University, Tiruchirappalli, in 1987, and the M.A.Sc. (Engg.) and Ph.D. degrees from the Department of Electrical and Computer Engineering, University of Toronto, Toronto, Canada, in 1993 and 1997, respectively. He worked for the Indian Space Research Organization for three years. He joined IIT Delhi in 1997, where he is currently a professor with the Department of Electrical Engineering. He was the Jai Gupta research chair professor for five years until September 2017. He holds three US and some Indian patents. He is a senior member of the IEEE. He has served in the technical program committees of prominent IEEE international conferences. $\mathrm{He}$ is currently serving as an editor of the IEEE TRANSACTIONS ON WIRELESS COMMUNICATIONS.

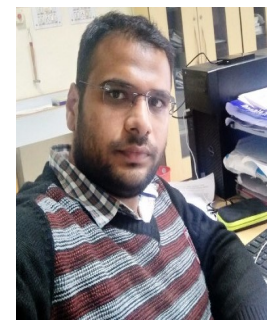

Kamal Agrawal (Graduate Student Member, IEEE) received the B.Tech. degree with honours in electronics and communication engineering from Gautam Buddha Technical University (formerly Uttar Pradesh Technical University), Lucknow, India, in 2010, and the M.Tech. degree in signal processing from the department of electronics and communication engineering, Netaji Subhas Institute of Technology (NSIT), University of Delhi, New Delhi, India, in 2016. He is currently working towards the Ph.D. degree from the department of electrical engineering, Indian Institute of Technology (IIT) Delhi, India. He was a visiting research student with the University College Dublin, Ireland, from April 2019 to July 2019, under the Erasmus+ ICM Research Program. His research interests include cooperative communications, cognitive-radio networks, aerial communication, energy harvesting, re-configurable intelligent surfaces and non-orthogonal multiple access techniques. 